\title{
Molecular Characterization of Brain and other Structures in Vertebrates and Invertebrates
}

\author{
Luisetto M*1, Gamal Abdul Hamid ${ }^{2}$, Naseer Almukhtar ${ }^{3}$, Ahmed Yesvi Rafa ${ }^{4}$, Behzad Nili Ahmadabadi ${ }^{5}$ \\ and Ghulam Rasool Mashori ${ }^{6}$
}

${ }^{1}$ Department of Applied Pharmacologist, Italy

${ }^{2}$ Department of Hematology Oncology, University of Aden, Yemen

${ }^{3}$ Department of Physiology, University of Babylon, Iraq

${ }^{4}$ Independent Researcher, Bangladesh

${ }^{5}$ Pharm D/PhD innovative Pharmaceutical product development specialist, USA

${ }^{6}$ Professor of Pharmacology, People University of Medical \& Health Sciences for Women, Pakistan

*Corresponding author: Luisetto M, Applied pharmacologist, European specialist lab medicine EC4 registered independent researcher, Italy.
Received Date: May 27, 2019

Published Date: May 30, 2019

\begin{abstract}
Comparative studies of brain and nervous system evolution - development and other structure in vertebrates and invertebrates and in their joining, link show some similarities or differences due by the gene expression. A wide scope of invertebrate immunocytes share with vertebrate phagocytic macrophages, phagocytosis, chemotaxis, tumorocidal activity and cytotoxic natural killer (NK) cells, and cytotoxicity in stress response or in transplantation. This fact can be useful to take in consideration in searching new therapeutic and pharmacological strategy of human disease like oncology, immunology, or degenerative pathology.
\end{abstract}

Keywords: Invertebrates; Vertebrates; Evolutionary; Pharmacology; Pathology; Molecular biology; Genetic regeneration; Immune system

\section{Introduction}

It is interesting to start this work observing some conservation of genes in evolution from invertebrates to vertebrates in some properties like organ or body part regeneration -restriction, nervous system development, immune system (adaptative vs innate), hormonal profile, DNA methylation properties opiates receptors profile, another. It Is possible to obtain useful information from this model in field of immune system disease, oncology or other degenerative pathology (like fibrosys et other)? Invertebrates model but also amphioxus and vertebrates show various abilities in regenerate some boy part and this is due by a specific gene code that regulate this kind of process. In pathology field the Fibrosis process is the formation of excess of fibrous connective tissue in an organ/tissue in a reparative or reactive kind of process: benign, or pathological. In response to injury, called scarring, and fibroma if fibrosis arises from a single cell line. Fibrosis acts to deposit a connective tissue, that can interfere with or totally inhibit the normal architecture and functionality of the underlying organ or tissue. (Fibrosis is the pathological state of excess deposition of fibrous tissue, and the process of connective tissue deposition in healing). Defined by the process of pathological accumulation of extracellular matrix (ECM) proteins, fibrosis results in scarring and thickening of the interested tissue, it is in essence an exaggerated wound healing response that interferes with normal organ functionality. (is similar to the scarring phenomena, involve stimulated fibroblasts laying down connective tissue, including collagen and glycosamino-glycans. The process start when the immune- cells (macrophages) release the soluble factors that stimulate the fibroblasts population. Pro-fibrotic mediator is the TGF beta, released by macro-phages and by any damaged tissue between surfaces named interstitium. Other mediators involved: CTGF, platelet-derived growth factor (PDGF), interleukin 4. These start the signal transduction pathways like s AKT/mTOR, SMAD pathways that ultimately lead to the proliferation - activation of the 
fibroblasts, whit deposit extracellular matrix into the surrounding connective tissue. This repair tissue is a very complex process, with tight regulation of the extracellular matrix (ECM) synthesis, degradation ensuring maintenance of the physiological normal tissue architecture. This process, even if necessary, can produce a progressive/ irreversible fibrotic response if tissue injury is severe (or repetitive), or if the wound healing response becomes a deregulated phenomenon (Figure 1).

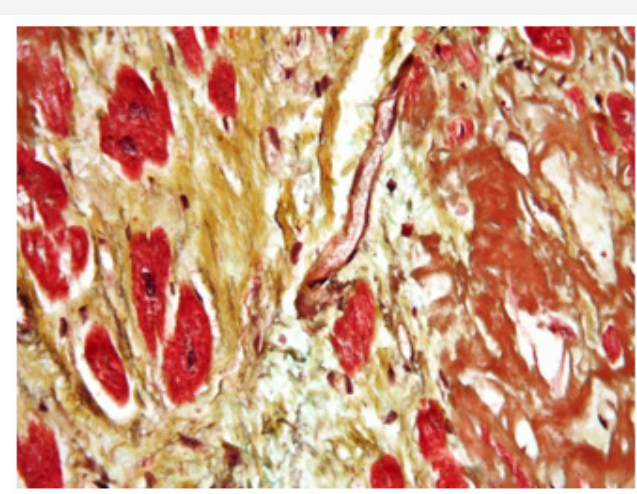

Figure 1: Fibrosis.

During the $20^{\text {th }}$ century, invertebrate zoology became one of the major fields of natural sciences, with great $t$ discoveries in medicine, genetics, paleontology, ecology and other sciences. Two of the most commonly studied model todays are invertebrates: the fruit fly Drosophila melanogaster and the nematode Caenorhabditis elegans. They were among the first life-forms to be genetically sequenced. This was facilitated by the severely reduced state of their specific genomes, but many genes, introns, and linkages have been lost. From article "Uncovering the truth of fibrosis": https://www.labnews.co.uk/features/uncovering-the-truth-offibrosis-29-07-2014/ is possible to verify that: "Despite its essential role in the normal wound healing process, fibrosis in humans is most often discussed in its pathological context - an irreversible, progressive and, ultimately, fatal disease. it can occur at any age, pathological fibrosis involving diverse organ -systems increases with age due to mechanisms that are only just being elucidated. Multiple biological pathways immediately become activated and synchronized to repair the damage. In the animal, there are many species that simply regenerate. Even complex body parts can be regenerated with full function and form following amputation or injury. Invertebrates such as the flatworm can regenerate the head from a piece of tail and the tail from the head.

Among vertebrates, fish can regenerate parts of the brain, eye, kidney, heart and fins. Frogs can regenerate limbs, tail, brain and eye tissue as tadpoles but not as adults; salamanders can regenerate limbs, heart, tail, brain, eye tissues, kidney, brain and spinal cord throughout life. This varying regenerative capacity is largely limited by each species' propensity to fibrosis following tissue injury or wounding.

In human adults, the wound repair process commonly leads to a non-functioning mass of fibrotic tissue (a scar). The inability to terminate this reparative response may underlie the progressive nature of fibrotic reactions in injured tissues. In contrast, early in human gestation, injured fo et al. tissues can be completely recreated, without fibrosis, in a process resembling regeneration. Fibrosis research is beginning to discover some of the pathways and elements involved in the repair mechanisms, comparing young and old, to one day hopefully have better treatments for these incurable set of diseases. Dysfunctional fibrotic healing can often cause lifelong disability. Human fibrotic disorders are estimated to contribute to $45 \%$ of all-cause mortality in the USA. The myofibroblast is a key effector cell in a wide range of fibrotic disorders and is primarily responsible for the extracellular matrix (ECM) synthesis and the tissue re-modelling in progressive fibrosis contests. In example in IPF is characterized by accumulating clusters of myofibroblasts in the lungs. We found that aged mice versus young mice, develop more persistent fibrotic response. Is associated with the emergence of senescent myo fibroblast cells that are resistant to apoptosis and mediated by a redox imbalance, triggered by sustained, elevated expression of NOX4 [NADPH (reduced form of nicotinamide adenine dinucleotide phosphate) oxidase-4], a reactive oxygen species (ROS)-generating enzyme, and a malfunction in the ability of the cells to induce the Nrf2 (NFE2-related factor 2) antioxidant response, a master regulator of antioxidant genes. Studies support the concept that loss of redox homeostasis in aging promotes the emergence/persistence of a senescent and apoptosis-resistant myofibroblast phenotype that sustains persistent/progressive fibrotic disorders.

We believe strategies that more directly target the source(s) of oxidative stress generation, for example with Genkyotex's GKT137831, may prove to be more specific and effective in comparison. Our recently published results not only provide preclinical evidence supporting targeting NOX4 as a viable strategy for therapeutic intervention in age-associated fibrotic disorders but also provide new insights into redox mechanisms that control profibrotic effects of fibroblast senescence". And Related cancer process: A neoplasm is a type of abnormal and excessive growth, named neoplasia, of a tissue. The growth of a neoplasm is uncoordinated with that of the normal surrounding tissue, and it persists growing abnormally, even if the original trigger is removed. This abnormal growth usually (but not always) forms a mass. When it forms a mass, it may be named a tumor. ICD-10 classify of neoplasms: benign neoplasms, in situ neoplasms, malignant neoplasms, and neoplasms of uncertain or unknown behavior.

Malignant neoplasms are also simply known as cancers. Prior to the abnormal growth of tissue, as neoplasia, cells often undergo an abnormal growth, such as metaplasia or dysplasia. metaplasia or dysplasia does not always progress to neoplasia. The term

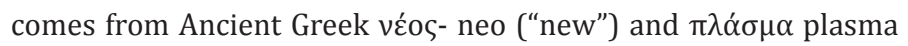
("formation", "creation"). Regarding this kind of process is possible to verify that there is an over activity of some cells that produce disease in an afinalistic process. But observing some conserved process since from invertebrates to some vertebrates other finalistic process are followed by this metazoan in example in regenerative 
ability of body part. So is crucial to verify in the evolutive pattern of metazoan conservation or deletion of some genes responsible of regeneration of body parts, immune system (innate od adaptative) and other process. Related the evolutive process and genetic the Analysis in example of the starlet sea anemone genome has emphasised the importance of sponges, placozoans, and choanoflagellates, being sequenced, in explaining the arrival of about 1500 ancestral genes unique to animals.

Invertebrates are animal that lacks a vertebral column, or backbone, in contrast to the cartilaginous or bony vertebrates. (about $90 \%$ of all living animal species are invertebrates). So are excluded fish, reptile, anphibious, birds and mammalians. First development in Precambrian period. Worldwide in distribution, they include animals as diverse as sea stars, sea urchins, earthworms, sponges, jellyfish, lobsters, crabs, insects, spiders, snails, clams, and squid. Apart from the absence of a vertebral column, invertebrates have little in common. All vertebrates are contained within a single phylum, the Chordata. (Phylum Chordata also includes the sea squirts and some other invertebrate groups.) Invertebrates are generally soft-bodied animals that lack a rigid internal skeleton for the attachment of muscles but often possess a hard outer skeleton (as in most mollusks, crustaceans, and insects) that serves, as well, for body protection.

Neurons differ in invertebrates from vertebrates. Invertebrates cells fire in response to similar stimuli as vertebrates to trauma, high temperature, or changes in $\mathrm{pH}$. Neurons have been identified in a wide range of invertebrate species, (annelids, molluscs, nematodes and arthropods). The earliest animal fossils appear to be of invertebrates. About 650-million year old interpreted as being early sponges. animals seems appeared much earlier, early as 1 billion years ago. Trace fossils like tracks and burrows found in the Tonian era indicate the presence of triploblastic worms, like metazoans. About 450 MYA, animals began diversifying, and many of the important groups of the invertebrates diverged from one another. Fossils of invertebrates are found in various types of sediment from the Phanerozoic. Fossils of invertebrates are commonly used in stratigraphy science (Figure 2 \& 3).

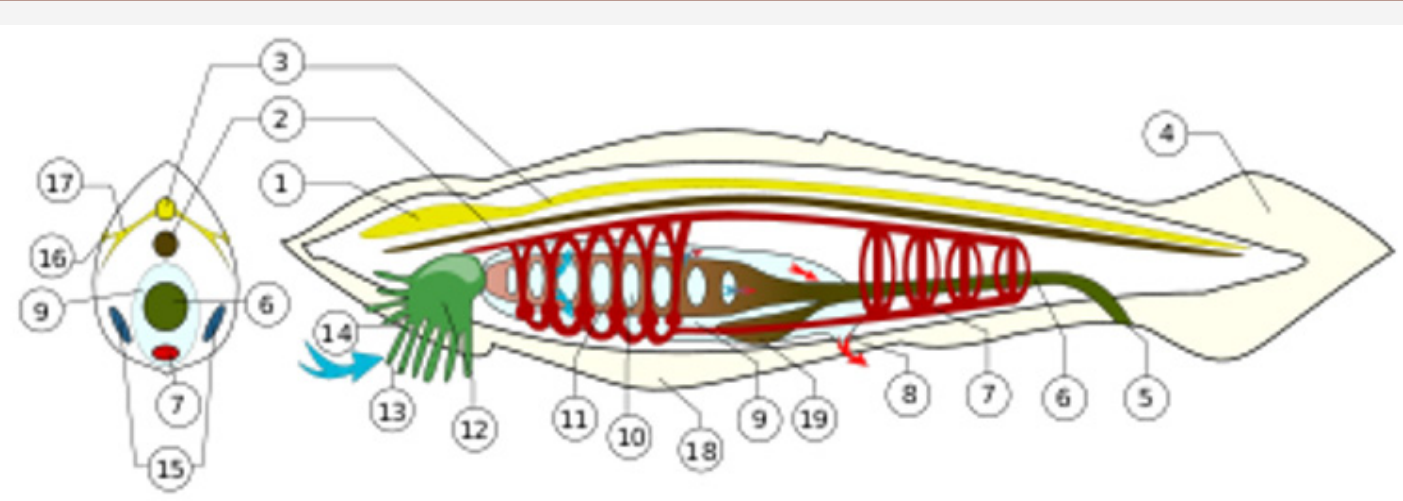

Figure 2:Branchiostoma lanceolatum, Anphioxus.

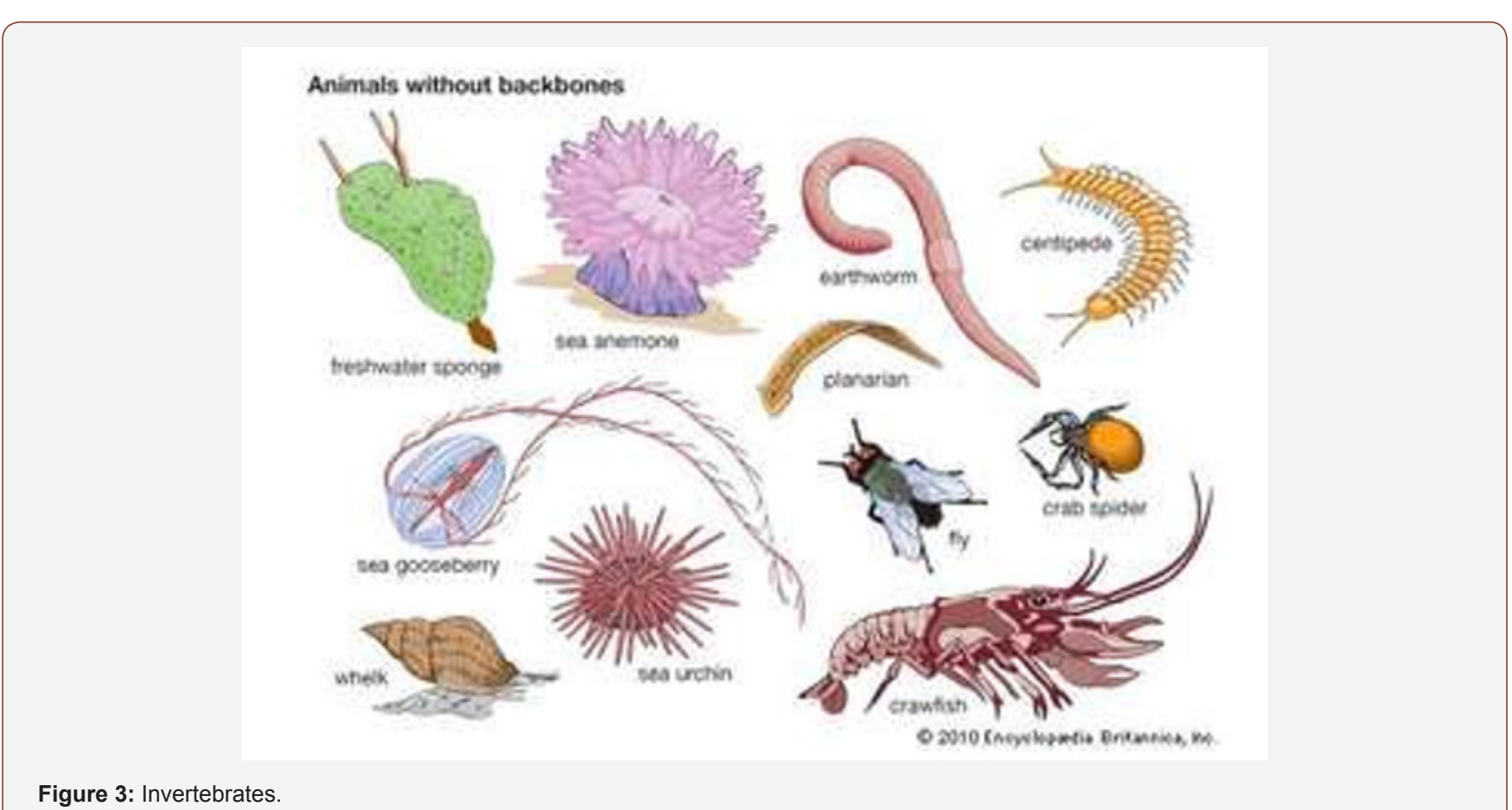


In article Worm-like Marine Animal Providing Fresh Clues About Human Evolution by Linda Holland: "Amphioxus is not widely known to the general public, but is gaining interest in scientific circles because of its position as one of the closest living invertebrate relatives of vertebrates. amphioxus split from vertebrates more than 520 million years ago, its genome holds tantalizing clues about evolution. Because amphioxus is evolving slowly-its body plan remains similar to that of fossils from the Cambrian time-the animal serves as an intriguing comparison point for tracing how vertebrates have evolved and adapted. This includes new information about how vertebrates have employed old genes for new functions. complicated vertebrate has not invented a lot of new genes to become complicated, Amphioxus shows us that vertebrates have taken old genes and recombined them, changed their regulation and perhaps changed the gene function. The human genome has only about 25 percent more genes than the amphioxus genome. During evolution, humans have duplicated genes for different functions. Such duplication has given to vertebrates a much larger "toolkit" for making various structures that are absent in amphioxus, including cells for pigment and collagen type II-based cartilage, roots of immunity. While vertebrates have 2 types of immune systems-innate, a first line of defense against pathogens, and adaptive, involving antibodies specific for particular pathogens-invertebrates like amphioxus have only the innate immune systems. In lanceolatum amphioxus, several of these innate immune genes have been independently duplicated many times over. It may be that with a $2^{\circ}$ line of defense, vertebrates, compared with invertebrates like amphioxus, are less reliant on innate immunity to ward off infection. The neural crest cells of vertebrates are an example of how "old" genes have acquired the new functions. In vertebrates, the neural crest cells

\section{acquired the new functions. In vertebrates, the neural crest cells}

migrate from the developing neural tube throughout the body, giving rise to such structures as pigment cells, cartilage of the head and a number of other kind of cell types.

Amphioxus has a brain and spinal cord and makes them using the same genes in the same way as vertebrates, amphioxus has no the neural crest cells And has the genes necessary for generating migratory neural crest cells; vertebrates have just put this genes together in new ways. So the amphioxus genome has really been a goldmine and will continue to be one in the years ahead." Related the evolution patterns of metazoans nervous system is possible to verify that: Neurons developed as specialized electrical signaling cells in multi-cellular animals, adapting the mechanism of action potentials present in the motile single-celled and colonial eukaryotes. Simple nerve nets seen in animals like the Cnidaria (jellyfish) evolved first, polymodal neurons whit a dual purpose in motor and sensory functions. Cnidarians can be compared to the Ctenophores (comb jellyfish), both jellyfish, but have very different nervous systems. Unlike Cnidarians, Ctenophores have neurons that use the electrochemical signaling system. (phylum Ctenophora was more ancient than that of Porifera (sponges), which have no a nervous system at all. According one theory: that the nervous system came about in an ancestor basal to all of these phylum but was lost in the Porifera.

Another theory: the nervous system arose independently twice, one basal to Cnidarians and one basal to Ctenophores. In the Bilateral animals - ventral nerve cords in invertebrates and dorsal nerve cords supported by a notochord in chordates, evolved in a central nervous system, around a central region, cephalization process (Figure 4).

\section{Anatomy of a Sponge}

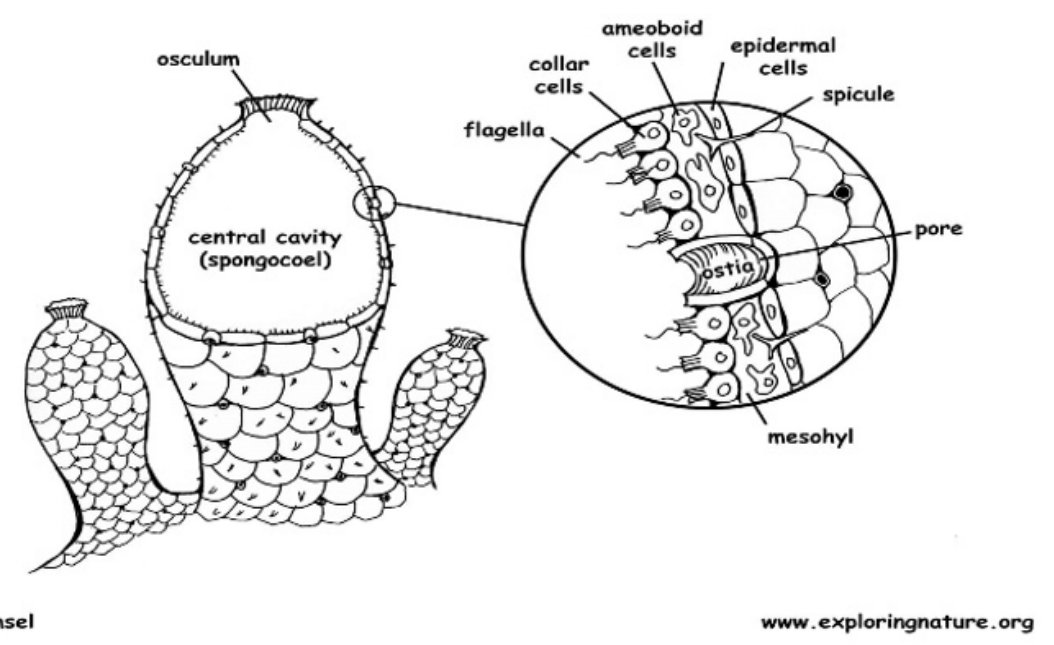

Figure 4: Anatomy of a sponge.

Sponges have no cells connected to each other by a synaptic junctions system, no neurons, no nervous system. They do have homologs of many genes that play a key roles in this synaptic function.
Studies showed that the sponge cells express a group of proteins that cluster together to form a structure resembling a postsynaptic density (the signal-receiving part of a synapse). Sponge cells do not 
show synaptic transmission, they do communicate with each other via calcium waves and other kind of impulses, that mediate simple actions such as whole-body contraction.

\section{Nerve nets}

Jellyfish, comb jellies, and the related animals have diffuse nerve nets than a central nervous system. In most of the jellyfish, the nerve net is spread more or less evenly across the intere body; in comb jellies it is concentrated near the mouth. The nerve nets is a sensory neurons that react to chemical, tactile, visual signals, motor neurons that activate contractions of the body wall, intermediate neurons that recognize patterns of activity in the sensory neurons and send signals to groups of motor neurons as a result. Groups of intermediate neurons are clustered into a discrete ganglia. The development of the nervous system in radiata is unstructured. Unlike bilaterians, radiata only have 2 primordial cell layers, the endoderm and the ectoderm. Neurons are generated from $\mathrm{f}$ ectodermal precursor cells, that serve as precursors for every other ectodermal cell type. Invertebrate Neural Induction: represents the initial step in the generation of the nervous system and begins with the segregation of neural and glial cells from other types of tissues. Experiments and research pertaining to neural induction are focused on invertebrates, specifically C. Elegans and Drosophila as well as vertebrates, specifically frogs. Invertebrates are much more powerful genetic systems due to how easily researchers can screen for they are looking for. This process is called forward genetics. Frogs on the other hand are not as good as the aforementioned invertebrates because of their slower life cycle and their tetraploid genes, which are much more difficult to manipulate. One benefit that studying vertebrates such as frogs brings are their big eggs, in which cellular changes can be better observed. The neurogenicregion of invertebrates begins in the ventro-lateral regions of the embryo. In the Drosophila Melanogaster, development start in the ventral furrow folds into the embryo interior. The invaginated cells become the mesoderm, and the neurogenic region becomes more ventral. The closure of the furrow creates a midline that will become the site of the neuro-genesis. The ectoderm of neuroblasts enlarge itself and squeeze away the epithelium layer through a delamination process. Delamination occurs in about 5 waves, named niches, each creating about 60 neuroblasts.

The neuro-blasts under-go cell division to produce a structure named "ganglion mother cell" (GMC). The GMC divides only once to produce neurons or glia.

\section{Vertebrate neural induction}

After the fertilization, the egg is polarized into a hemisphere. The hemisphere, at the top of the egg (the neurogenic region) has smaller cells than the rest of the egg. Following this, a Blastula is formed after the egg under-goes multiple division, with a blactocoel being the outcome. This differs from the blastula: the tiny pocket or space that is created. Then the gastrula is formed via the process of gastrulation which leads to the creation of the neurula. And, the neurogenic regions turns in to the neural plate, precursor to the neural tube, later becomes the brainstem. The creation of the neural- tube occurs once the neural plate folds inwards. Along with the neural tube, the neural crest is also created at this time, and it is the space in between the neural tube and ectoderm. The neural crest produces the neurons and glia that lie outside of the CNS, like the peripheral nervous system. Molecular Signaling of Neural Induction, BMP signaling pathway, The Wnt signaling pathway many major pathways which ultimately regulate gene expression.

2 signaling cascades which appear to effect gene expression early on are the BMP and Wnt signaling pathway.

BMP pathway starts with BMPs binding to a receptor composed of type 1 and 2 subunits. This receptor is a major determiner in setting up the epidermal cells. When BMP is NOT present the removal of the animal cap leads to the creation of neurons. IF BMP present, the removal of the animal cap leads to the epidermal cells. When the receptor is bounded, the type 2 subunit phosphorylates the type 1 subunit. The phosphorylation of the type 1 subunit causes further the phosphorylation of the RSMAD protein. These phosphorylated RSMADs for a complex with coSMADs forming a RSMAD: coSMAD complex (then moves into the cell nucleus). the complex binds to DNA sequences called BMP response elements which are present in the promotor regions of genes. This initiates the process of transcription. Wnt and Shh pathway interaction. The Wnt process begins when the Wnt protein binds to a receptor called Frizzled. When Wnt is bound to the receptor, a protein $\beta$-catenin binds with many other proteins. A protein named Disheveled prevents degradation of the formed complex only when Wnt is bound to Frizzled.

As $\beta$-catenin accumulates, some of it moves into the nucleus where it complexes with TCF. The formed TCF $\beta$-catenin complex binds to DNA and activates the transcription. Nerve cords. A rod-shaped body contains a digestive system running from the mouth at one end to the anus at the other. Alongside the digestive system is a nerve cord with a brain at the end, near to the mouth. Nervous system of a bilaterian animal, in the form of a nerve cord with a "brain" at the front. The majority of existing animals are bilaterians, with left and right sides that are approximate mirror images of each other. This are thought to have descended from a common wormlike ancestor that appeared 550 million years ago. The bilaterian body form is a tube with a hollow gut cavity running from mouth to anus, and a nerve cord with an especially large ganglion at the front, named the "brain". Area of the human body surface innervated by each spinal nerve Even mammals show the segmented bilaterian body plan at the level of the NS. The spinal cord contains series of segmental ganglia, each giving rise to motor and sensory nerves that innervate a SPECIFIC portion of the body surface and underlying musculature. On the limbs, the layout of the innervation pattern is more complex, but on the trunk it gives rise to a series of narrow bands.

The top 3 segments belong to the brain, giving rise to the forebrain, midbrain, and hindbrain. Bilaterians can be divided, based on events that occur very early in embryonic development, into 2 superphyla: protostomes and deuterostomes. Deuterostomes 
include vertebrates like echinoderms and hemichordates (mainly acorn worms). Protostomes include arthropods, molluscs, and numerous kind of worms. There is a fundamental difference between the 2 groups in the placement of the NS in the body: protostomes possess a nerve cord on the ventral (usually bottom) side of the body, whereas in deuterostomes the nerve cord is on the dorsal (usually top) side. Various aspects of the body are inverted between the 2 groups, including the expression patterns of several genes that show dorsal-to-ventral gradients. Some researcher anatomists now consider that the bodies of protostomes and deuterostomes are "flipped over" with respect to each other, first proposed by G. Saint-Hilaire for insects in comparison to vertebrates. Thus insects, have nerve cords that run along the ventral midline of the body, while all the vertebrates have spinal cords that run along the dorsal midline. Recent molecular data from different protostomes and deuterostomes reject this kind of scenario. In frogs, involuting mesodermal cells of the involuting marginal zone release Chordin, Noggin, and Follistatin to inhibit BMP, causing the induction of neural tissue.

Nervous system development is quite similar among thousands of different species, demonstrating an evolutionary connection of some kind. One of the primary examples of this is orthologs. Orthologs are any 2 or more homologous gene sequences found in different species that are related by linear descent. As stated previously, BMP inhibits neural differentiation in vertebrates. Drosophila Melanogaster possesses a molecule known as dpp, which also inhibits neural differentiation in a similar way. Sonic hedgehog (shh) is the morphogen of vertebrates that induces neural crest development by inhibiting BMP. Drosophila has sog, which inhibits dpp, causing similar results.

\section{Homeobox (HOX) genes in invertebrates}

After the formation of the regionally specified anterior-posterior axis, genes must be turned "on" to form unique structures in specific regions. This is orchestrated via the homeobox (Hox) transcription factor signaling pathway. First identified in Drosophila by Edward Lewis who won a Nobel Prize for the discovery.

Homeobox genes are organized in Drosophila along 2 complexes [Antennapedia complex (ANT-C) and Bithorax Complex (BX-C)]. 8 genes are organized in the cluster based upon anterior-posterior expression.

Homeobox proteins are transcription factors composed of a highly conserved 60 amino acid protein sequence present in many kind of organisms. Homeobox proteins bind to specific sequences of the DNA of genes to regulate their related expression. HOX clusters like the ones in Drosophila have been identified in vertebrates. Even if there are more HOX genes in vertebrates, the position of the HOX genes in relation to its expression along the A-P axis is conserved among species. Hox genes in Drosophila Rhombomeres in Humans Homeobox Genes in Vertebrates (Rhombomeres). Studies into the role of HOX genes in vertebrate NS development has come from studies about hindbrain formation. The hindbrain forms a segmented pattern reminiscent of the segments within the Drosophila embryo. In vertebrates, these segments of the hindbrain are referred to as rhombomeres. The rhombomeres are numbered from the anterior most unit, $r 1$, which is just posterior to the midbrain, to the posterior most unit, r8, at the border between the hindbrain and the spinal cord. Each rhombomere gives rise to a unique set of motor neurons that controls different muscles in the head. $r 2$ and r3 make the trigeminal motor neurons that innervate the jaw. The facial nerve motor neurons are mainly produced in $\mathrm{r} 4$ and the abducens motor neurons are produced in r5. Loss of the Hoxa1 gene in mice results in a complete loss of $\mathrm{r} 5$ and a reduction in $r 4$. This causes severe shrinking of the facial nerve and a total loss of the abducens nerve.

\section{Nerve cords}

A rod-shaped body contains a digestive system running from the mouth at one end to the anus at the other. Alongside the digestive system is a nerve cord with a brain at the end, near to the mouth. Nervous system of a bilaterian animal, in the form of a nerve cord with a "brain" at the front. The vast majority of existing animals are bilaterians, meaning animals with left and right sides that are approximate mirror images of each other. All bilateria are thought to have descended from a common wormlike ancestor that appeared in the Ediacaran period, 550-600 million years ago. The fundamental bilaterian body form is a tube with a hollow gut cavity running from mouth to anus, and a nerve cord with an especially large ganglion at the front, called the "brain". Area of the human body surface innervated by each spinal nerve. Even mammals show the segmented bilaterian body plan at the level of the NS. The spinal cord contains series of segmental- ganglia, giving rise to motor and sensory nerves that innervate a portion of the body surface and musculature. On the limbs, the layout of the innervation is complex, on trunk it gives rise to a series of narrow bands.

The 3 segments belong to the brain, giving rise to the forebrain, midbrain, and hindbrain. Related joining link between invertebrates to vertebrates Amphioxus is generally considered the joining link between vertebrates and invertebrates, or the most near ancestor of vertebrates. The group of amphioxus and the tunicates are classified as chordates because present a dorsal axile structure of support: the dorsal chord.

This structure is replaced in vertebrates whit vertebral column that with the dorsal nervous system. Is a fundamental tract in this group. Filogenesys and ontogenesys of this kind of joining link can be usefull instrument to this work.

\section{Nervous system}

In common with vertebrates, amphioxus have a hollow nerve cord running along the back, pharyngeal slits and a tail that runs past the anus. like vertebrates, the muscles are arranged in blocks called myomeres.

Unlike vertebrates, dorsal nerve cord is not protected by bone but by a notochord made up of a cylinder of cells that are closely packed to form a toughened rod. The lancelet notochord, unlike the vertebrate spine, extends into their head. This gives the subphylum 
its name (kephalē means 'head'). The nerve cord is only slightly larger in the head region than in the rest of the body, so lancelets do not appear to possess a real true brain structure. The developmental gene expression and transmission electron microscopy technique indicate the presence of a diencephalic forebrain, a possible midbrain, and a hindbrain, recent research involving a comparison with vertebrates indicates that vertebrate thalamus, pretectum, and midbrain domains jointly correspond to a single amphioxus region, Di-Mesencephalic primordium (DiMes).

\section{Lancelets amphioxus other systems}

Lancelets have no a real respiratory system, breathing solely through the skin, which consists of a simple epithelium like any respiration occurs in the "gill" slits, which are devoted to feeding. The circulatory system does resemble that of primitive fish, but much simply, not include a heart. There are no blood cells system, no haemoglobin. The excretory system consists of segmented "kidneys" containing protonephridia instead of nephrons, and quite unlike those of vertebrates. Also unlike vertebrates, there are numerous, segmented gonads. The same other characteristics of like regenerative properties of some body parts and the related genes involved (invertebrates- some vertebrates). This property differ a lot between species from species. Some invertebrates can regenerate the full body also strating from a part of this. This property also can variates in the different vital cycle or age of the animal involved. Anphybious, in vertebrates group, can regenerate complete and functional organs and tissue, in mammalians there is a limited capacity (regeneration of liver after a damage).

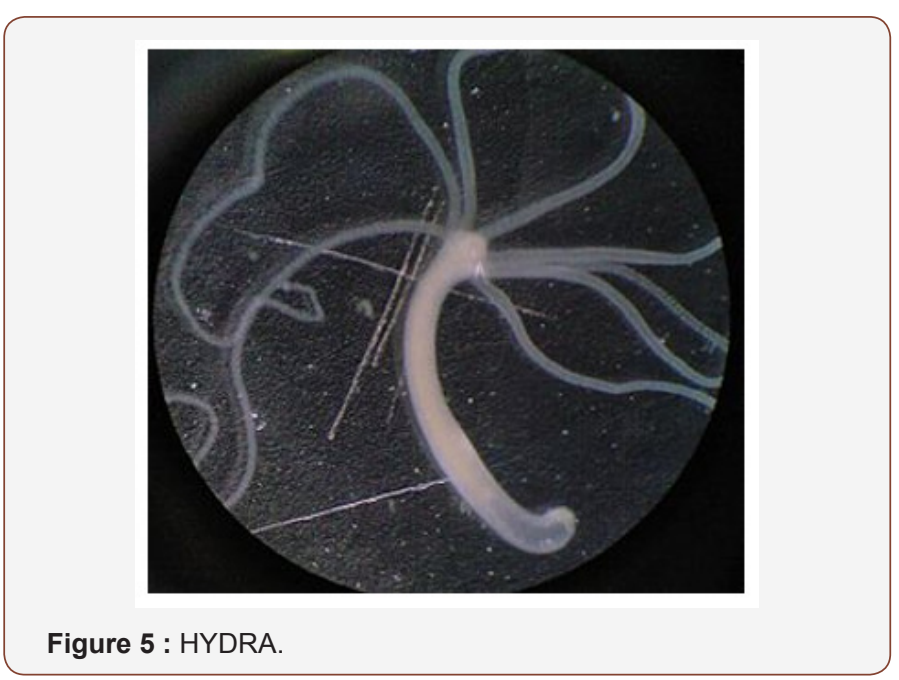

It Is important for this study to verify the reason why mammalians lose the property to regenerate limbs if damaged. First genes activates in regeneration in different animals seems of a common origin.(invertebrates- vertebrates). In advanced phases of regeneration the genes involved are more species specific or tissue specific. Is very interesting the fact that in regenerative process are involved the genes of immuitary responce of inflamation process. (not only a defence systems). In example the immunitary systems of HYDRE in the early stage of the inflamatory responce are activated genes similar to the one involved in macrophages and neutrofile activation in mammalians. Crucial genes involved in regeneration and in surviving capacity seem to be conserved in evolution pattern of Vertebrates and invertebrates. A better understanding of this kind of phenomena can be useful in some pathology(in example in surplus of fibrosis but in many other condition). Hydre are little sea invertebrates, whit high regeneration ability, less efficient are planarie, due by high level of stem cell in all their body (Figure 5).

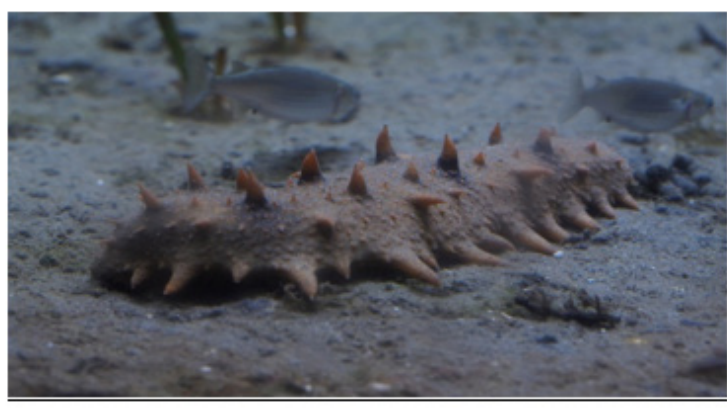

Figure 6: Apostichopus japonicus.

Also Echinoderms like Apostichopus japonicus can regenerate itys internal orgnans after damages (a defence property) (Figure 6).

Notall animal present the same ability of regeneration: lizard can regenerate only the tail and in vertebrates group only anphybious can regenerate complete anatomically tissue with functionality. In mammalian only the liver can be regenerate but whit a loss of its anatomical structure (lobi but whit a substantial functionality. Similarity and difference in evolution of species show us the link but also the diversify. A common gene set to hydre, planarie, mouses, (and the expressione levels change in the first fases). Or increase or reduced expression (involved in cell comunication, DNA repair, trascription and the other. Involved in methabolic process, cells adesion, immune response. This facts show a common link between invertebtares to vertebrates since mamalians. Differences are present in later fases of regeneration with involving of more eterogeneous genes.(more species and tissue related). Also observing the brain evolution: the most ancient example of complex brain start form camrbrian period. Telencefalous philogenesis: in vertebrates originates from ectoderm (external germ in first embrional phases). Instead cordates and invertebrates present a more linears nervous systems withou the cerebral region see in vertebrates. But even this differences similarity are present: signal system center of central nervous system that can be considered the bases for vertebrates evolution. (secondary organizators).

Anterior neural crest, intra-talamic limitanting zone and istmic organizator also in in sea warms Saccoglossus kowalevskii. Genes Fgf8/17/18, sfrp1/5, hh and wnt1 are expressed in emicordates ectodermaswell as in vertebrates. (an ancient systems of regulation). During evolution this structure degenerated in amphioxus and ascidia but conserved in emicordates and vertebrates. taxonomists place the lampreys and the hagfish in the subphylum Vertebrata of the phylum Chordata, which also includes the invertebrate subphyla Tunicata (sea-squirts) and the fish-like Cephalochordata (lancelets or Amphioxus). Recent molecular -morphological phylogenetic studies place the lampreys and hagfish in the superclass Agnatha or 
Agnathostomata (both meaning without jaws). The other vertebrate super-class is Gnathostomata (jawed mouths), includes the classes Chondrichthyes (sharks), Osteichthyes (bony fishes), Amphibia, Reptilia, Aves, and the Mammalia. Notochord is a flexible tubular structure present in all cordates embryons (ventral position related neural tube). In cefalocordates and in primitive vertebrates this structure all adult life time as sustaining structure.

In urocordates is present in larval stadio, and in vertebrates is sobstituted by vertebral column. Vestigial notocord are present i nel nucleous polpose of intervertebral discus, but not in vertebral body. In humans, at 4 year, all residual of notocord are sobstituted by similar condrocites population. Urocordates (primitive vertebrates) present similar charctheristics in nervous systems organization. Other interesting example can be: mammalian telomeric structure: T-loop that protect Histon H3 H4 are high conserved in eucariotes.

From invertebrates to vertebrates 2 duplication genaoma event s: cluster hox quadruplicate. And the Globine genes in vertebrates 800 mil years ago originated myoglobin, alfa and beta globine by duplication process. (fetal hemoglobin need more 02 affinity for gestation periods). But many other examples can be introduced in this works to better understand the global research hypotesys submitted in discussion.

\section{Material and Methods}

With an observation approach relevant bibliography and reference are analyzed to produce a global. Conclusions related to the aim of this work. All the references are present in biomedical databases like PUBMED and other resourcers. After this phases and experimental project will be submitted to the researcher.

\section{Result}

From literature: Kammermeier $\mathrm{L}$ et al: "Recent genetic studies on embryonic brain development in the fly Drosophila melanogaster together with investigations on early morphogenesis and patterning in the embryonic brain of the mouse revealed developmental mechanisms that are strikingly similar in insects and mammals. The homeotic (Hox) genes are expressed in a virtually co-linear antero-posterior pattern in the developing posterior brain of insects and mammals, where they are required for the specification of segmental neuronal identity. The otd/Otx cephalic gap genes are expressed in the anterior brain of insects and mammals and are of central importance for its formation because in both phyla loss of otd/Otx2 causes the loss of the entire rostral brain. Specific Pax genes are involved in numerous aspects of brain development in both phyla. These developmental genetic findings reveal a striking evolutionary conservation of cephalic gap gene, homeotic gene, and Pax gene action in embryonic brain development that extends beyond gene structure to encompass patterned expression and function. This comparative evidence indicates that the genetic programs which direct embryonic brain development are remarkably conserved and lends further support to the hypothesis that a common molecular bauplan underlies brain development in invertebrates and vertebrates. In consequence, it seems increasingly likely that both modern brain types share their evolutionary origin in a common ancestral bilaterian brain which was established before the protostome-deuterostome divergence over 600 million years ago" [1].

Nam J1, Nei M: "It has been known that the conservation or diversity of homeobox genes is responsible for the similarity and variability of some of the morphological or physiological characters among different organisms. To gain some insights into the evolutionary pattern of homeobox genes in bilateral animals, we studied the change of the numbers of these genes during the evolution of bilateral animals. We analyzed 2,031 homeodomain sequences compiled from 11 species of bilateral animals ranging from Caenorhabditis elegans to humans. Our phylogenetic analysis using a modified reconciled-tree method suggested that there were at least about 88 homeobox genes in the common ancestor of bilateral animals. About 50-60 genes of them have left at least one descendant gene in each of the 11 species studied, suggesting that about 30-40 genes were lost in a lineage-specific manner. Although similar numbers of ancestral genes have survived in each species, vertebrate lineages gained many more genes by duplication than invertebrate lineages, resulting in more than 200 homeobox genes in vertebrates and about 100 in invertebrates. After these gene duplications, a substantial number of old duplicate genes have also been lost in each lineage. Because many old duplicate genes were lost, it is likely that lost genes had already been differentiated from other groups of genes at the time of gene loss. We conclude that both gain and loss of homeobox genes were important for the evolutionary change of phenotypic characters in bilateral animals" [2].

Susanne Dreborg et al: "The opioid peptides and receptors have prominent roles in pain transmission and reward mechanisms in mammals. The evolution of the opioid receptors has so far been little studied, with only a few reports on species other than tetrapods. We have investigated species representing a broader range of vertebrates and found that the 4 opioid receptor types (delta, kappa, mu, and NOP) are present in most of the species. The gene relationships were deduced by using both phylogenetic analyses and chromosomal location relative to neighboring gene families in databases of assembled genomes. The combined results show that the vertebrate opioid receptor gene family arose by quadruplication of a large chromosomal block containing at least other gene families. The quadruplication seems to coincide with, and, probably resulted from, the 2 proposed genome duplications in early vertebrate evolution. We conclude that the quartet of opioid receptors was already present at the origin of jawed vertebrates 450 million years ago. A few additional opioid receptor gene duplications have occurred in bony fishes. Interestingly, the ancestral receptor gene duplications coincide with the origin of the 4 opioid peptide precursor genes. Thus, the complete vertebrate opioid system was already established in the first jawed vertebrates. chromosome G protein An ortholog to one of the intron less NPBW receptors was identified in the elephant shark genome. Fragments from 3 putative opioid receptors have been cloned in thresher shark 
(Alopias vulpinus), and some fragments of opioid-like receptors from a jawless fish, the Pacific hagfish (Eptatretus stoutii), have also been cloned. Our analyses are not able to determine which receptor type the cloned hagfish sequence corresponds to (data not shown). The genome of the sea lamprey (Petromyzon marinus), a species from another lineage of jawless fishes, has been sequenced but is only assembled into small unlinked contigs and our blast searches only gave partial sequences (data not shown). These sequences did not contain enough information to be assigned to specific opioid receptor types. 2 intronless NPBW receptor like sequences were found in the sea lamprey genome. We were not able to find any opioid receptor sequences in the Florida lancelet or the tunicate genome databases. A report of a mu receptor sequence obtained from the blue mussel (Mytilus edulis) by RT-PCR that displayed 95\% nucleotide sequence identity to the human mu receptor has been published. The most likely explanation for this extraordinary degree of sequence identity between a mollusc and a mammal is either horizontal gene transfer or contamination. Until this has been resolved, we prefer not to include the claimed blue mussel mu receptor sequence in our phylogenetic trees [3]."

Celine J Vivien et al: "There are 64,000 living species of vertebrates on our planet and all of them have a heart. Comparative analyses devoted to understanding the regenerative potential of the myocardium have been performed in a dozen vertebrate species with the aim of developing regenerative therapies for human heart disease. Based on this selection of animal models, important insights into the evolutionary conservation of regenerative mechanisms have been gained. we survey cardiacregeneration studies in diverse species to provide an evolutionary context for the lack of regenerative capacity in the adult mammalian heart. Our analyses highlight the importance of cardiac adaptations that have occurred over hundreds of millions of years during the transition from aquatic to terrestrial life, as well as during the transition from the womb to an oxygen-rich environment at birth. We also discuss the evolution - ontogeny of cardiac morphological, physiological and metabolic adaptations in the context of heart regeneration. The evolution of a multi-chambered, high-pressure, closed circulatory system has necessitated a number of important morphological and physiological adaptations. That have occurred over millions of years of evolution during the transition from water to land and occur over days to months during the developmental transition from foetal to post-natal life. we discuss these evolutionary and developmental adaptations in the context of heart regeneration. Morphological adaptations Several different heart forms are found throughout the animal kingdom from the simplest heart tubes in flies to the more complex multi-chambered hearts of fish, amphibians and mammals. These adaptations have enabled the successful transition from water to terrestrial life. All vertebrates possess a closed and multi-chambered heart and cardiac morphogenesis progresses along a highly conserved developmental path involving formation of a linear tube, looping of the tube and finally formation of the chambers. With regards to the final morphology of the heart, fish and amphibian larvae have a 2-chambered heart composed of one atrium and 1 ventricle. Our findings suggest that cardiac regenerative potential correlates with a low-metabolic state, the inability to regulate body temperature, low heart pressure, hypoxia, immature cardio-myocyte structure and an immature immune system. Regeneration is a trait that exists within different phyla, orders and species in the animal kingdom. Invertebrates such like planarians, crustaceans, cnidarians, echinoderms and insects, are known to have a strong global regenerative potential. regenerative capacity can vary considerably even in a given order. Even among the highly regenerative planarian flatworms, which are capable of regenerating large portions of the body including the head, 5 species with a more limited regenerative ability are found. Procotyla fluviatilis has a restricted ability to replace missing tissues due to changes in the Wnt signalling pathway in this planarian species. regenerative capacity is also differentially distributed in vertebrates. Newts, axolotls and zebrafish are well-known for their abilities to replace entire limbs, fins and other body parts following amputation. Regenerative capacity is restricted in many mammals. regenerative capacity in non-mammalian vertebrates seems to rely on the re-activation of highly conserved developmental signaling pathways, it is unclear whether the lack of regenerative potential in mammals is due to an improper re-activation of these developmental pathways or whether it is due to intrinsic cellular changes that prevent the cells from responding in a regenerative manner to the developmental cues (or both) non-cardiomyocyte populations in the heart also regenerate after myocardial damage and they are required to support cardio- myocyte proliferation and remodelling. The endocardium and epicardium are activated following injury and reexpress developmental genes, which contribute to cardio-myocyte proliferation, angiogenesis and fibroblast differentiation. studies highlight the potentially important roles of non-myocytes in the regenerative response but functions of these other cell types in conferring regenerative capacity remain understood. Zebrafish larvae. To date, only one approach has been used to study cardiac regeneration during early-embryonic stages of zebrafish development. In this research, cardio myocytes were genetically ablated in zebrafish larvae using a method that enabled cardiomyocyte-specific and temporally controlled depletion of ventricular cardio- myocytes. zebrafish embryos are able to fully regenerate the damaged myocardium in only 4 days after this type of injury but the mechanism of regeneration differs from the adult heart. The authors of this were described a global proliferative response in the regenerating ventricle, with proliferating myocytes detected in the atria, as well as the ventricle. in addition to cardio-myocyte proliferation, genetic fate-mapping studies revealed that cardiomyocytes from the atrium were able to migrate into the damaged ventricle, proliferate and regenerate the ventricular myocardium. Cardio-myocytes from the atrium underwent an atrial-toventricular trans-differentiation (or reprogramming) process, which required the activation of Notch signaling in the atrial endocardium. To date, this trans differentiation process has only been described in zebrafish larvae but this finding suggests that the same organism can employ multiple mechanisms of cardiac regeneration depending on the developmental stage. Giant Danio 
(Devario aequipinnatus), Goldfish (Carassius auratus) and Polypterus (Polypterus senegalus). The Giant Danio and Goldfish are also teleosts belonging to the Cyprinid family. The regenerative response to only one type of injury, namely cauterisation using a hot probe to burn the ventricular myocardium, has been documented in these 2 fish models. In the Giant Danio, cauterisation induces the destruction of $30 \%$ of the ventricle, whereas this mode of injury induces damage of $11 \%$ of the ventricle in Goldfish. Both of these fish mount a robust regenerative response following cardiac injury and they are able to reconstitute $\sim 96 \%$ of the myocardium in 40-50 days. The cardiac regenerative response in the Giant Danio is similar to the regenerative response of adult zebrafish following cryo injury and is associated with the presence of inflammatory cells, collagen deposition and the establishment of a transient fibrotic scar that is progressively replaced with new myocardial tissue. The Giant Danio is twice as large as the zebrafish and Goldfish is even bigger. As a consequence, these models may be more suitable to the development of more physiologically relevant cardiac injury models such as coronary artery ligation to induce MI, which would facilitate direct comparative studies between fishes and mammals. Due to their larger size, these fish models could also be useful for comparative analyses of the cardiac regenerative response to ventricular amputation or cryoinjury at different developmental stages in teleosts. Cardiac regeneration has also been assessed in the ancient fish P. senegalus, which is a member of the Polypteridae family. The last common ancestor between Polypterus and teleosts lived $\sim 400$ million years ago. Ventricular resection or stab injuries were performed on the heart of juvenile Polypterus. Interestingly, 7 days after injury, cardio-myocyte proliferation was seen in the injured area. As in zebrafish, retinoic acid was released from the epicardium and endocardium after cardiac injury in Polypterus. These results suggest that the epicardial and cardio-myocyte proliferative responses to cardiac injury are highly conserved by evolutionarily distant species capable of heart regeneration. The rates of cardio-myocyte proliferation observed in Polypterus were lower than the rates observed in zebrafish. the true extent of cardiac regeneration in Polypterus is unclear because this study did not provide quantitative analyses of the extent of tissue removal and fibrosis after injury.

Medaka (Oryzias latipes). Medaka is a teleost fish that is approximately the same size as zebrafish. medaka belongs to the Beloniformes order, which diverged from the Cypriniform order during the early Triassic around 215 million years ago. Interestingly, in contrast to zebrafish, Giant Danio and Goldfish, medaka lack cardiac regenerative ability. Mechanical resection of the apex of the heart results in removal of $20 \%$ of the medaka ventricle, which is a similar injury size to zebrafish apical resection models. After amputation, a blood clot forms at the injury site and is subsequently replaced by a fibrotic scar. The initial events following ventricular amputation in medaka are similar to the first events occurring in the zebrafish model, with the exception of a lack of epicardial activation of retinaldehyde dehydrogenase 2 (raldh2), which is required for synthesis of retinoic acid. Contrary to zebrafish, no difference in cardio- myocyte proliferation was seen and the collagen area expanded instead of regressing. The absence of retinoic acid signalling from the epicardium could account for the lack of regenerative capacity in medaka. Regeneration studies performed in teleost fish demonstrate that fish belonging to the Cyprinid family have a widely conserved capacity to regenerate the myocardium after damage whereas the medaka, an Adrianichthyidae family member, does not possess cardiac regenerative abilities. It appears that cardiac regenerative capacity is not conserved across all teleost fish. New studies in others fish families are necessary to clearly understand the evolutionary basis for regenerative differences, as well as the evolutionary pressures that have driven the acquisition and maintenance of regenerative capacity in some fish and the loss of regenerative capacity in others. it would be interesting to examine the capacity for cardiac regeneration in lungfish, which represent an important evolutionary intermediate between aquatic and terrestrial vertebrates. Lungfish are known to be able to fully regenerate their fins following amputation but it is unknown whether a similar regenerative response occurs following cardiac injury. Amphibians can be separated into 3 orders, urodele (newt, salamander and axololt), anuran (frogs and toads) and apoda (caecilians). Urodele amphibians have a functional tail in adult life, whereas anuran amphibians lose their tail after metamorphosis and Apoda are limbless with a worm-like form. Anuran and urodele amphibians are able to regenerate several tissues and organs such as limbs, retina and nerve tissue. They are characterised by their 2 different life environments because they live in water as an aquatic (axolotl) or larval (tadpole) form; or on land as a terrestrial form (frogs, salamanders and terrestrial axolotls).

Neoteny, which is the maintenance of larval/aquatic characteristics throughout life, naturally occurs in urodele amphibians. urodele and anuran amphibians are useful models to explore the mechanisms of cardiac regeneration in aquatic and terrestrial tetrapod vertebrates. In amphibians, heart regeneration occurs during aquatic life but, to date, cardiac regeneration has not been documented in terrestrial amphibians. Cardiac regeneration in mammals appears to be restricted during a defined earlydevelopmental period during embryonic and early-neonatal life. Immune system. Several similarities between non-mammalian vertebrates and young mammals have been noted with regards to the absence of a more sophisticated immune system compared with adult mammals in terms of specificity, speed of onset and adaptive memory. non-mammalian vertebrates lack specialized proteins such as immunoglobulins and the neonatal mammalian immune system has impaired pro-inflammatory functions. Urodele amphibians possess innate immunity but lack a complete adaptive immune system.3 Experiments performed on limb regeneration suggest that this less sophisticated adaptive immune system is involved in the regulation of regenerative ability in these species.

In anuran amphibians, tadpole tails are able to regenerate but this ability is lost during the refractory phase, which is associated with changes in the immune response to injury. 
Immunosuppression restored regenerative ability during the refractory period in the anuran amphibian. Interestingly, macrophages are required for appendage regeneration in zebrafish and urodele amphibians, as well as heart regeneration in neonatal mice. Macrophages are present within the infarcted area in both adult and neonatal mice in response to injury but the neonatal heart expands a population of resident cardiac macrophages, which differ from adult macrophages in terms of their origin and immunophenotype. Macrophages influence neovascularization during neonatal heart regeneration but have no direct impact on cardio-myocyte proliferation in this model. phylogenetic changes in regenerative capacity are associated with evolutionary changes in the activity of the immune system and there seems to be an inverse relationship between regenerative capacity and the development of a mature immune system. These evolutionary adaptations may have resulted from pressures that permitted the development of adaptive immune mechanisms that promoted animal survival in the face of infectious diseases but resulted in a loss of reparative potential because of excessive inflammation following tissue injury.

Blood clotting, inflammation and fibrosis. As previously described, depending on the animal model, heart injury can result in regeneration or permanent scar formation. Blood clotting is an early feature of the wound healing response and is associated with either regeneration or fibrotic healing depending on the animal model. In some example, clotting factors provide signals that are required for regeneration. in salamander lens regeneration and in murine liver regeneration, it appears that coagulation and other blood cell-dependant mechanisms, such as platelet activation, provide important signals for cell-cycle re-entry and regeneration. Interestingly, the newt lens regenerates, whereas the closely related axolotl lens cannot. Clotting factors, such as thrombin, have been implicated in the differential regenerative capacity of the lens in these two amphibian models. Thrombin is also known to induce cell-cycle re-entry in cultured newt myocytes. Other growth factors released from thrombocytes at the site of injury, such as plateletderived growth factor BB isoform (Pdgf-BB), play important roles in epicardial cell function and coronary vessel formation during zebrafish heart regeneration. blood clotting is an important early event in the cardiac regenerative response. blood clotting also occurs following coronary artery ligation in adult mammals, where it is associated with early-wound healing in the setting of fibrotic repair. Given that clotting is associated with both tissue repair and regeneration, it is unclear how clotting factors specifically drive regenerative processes instead of permanent scar formation.

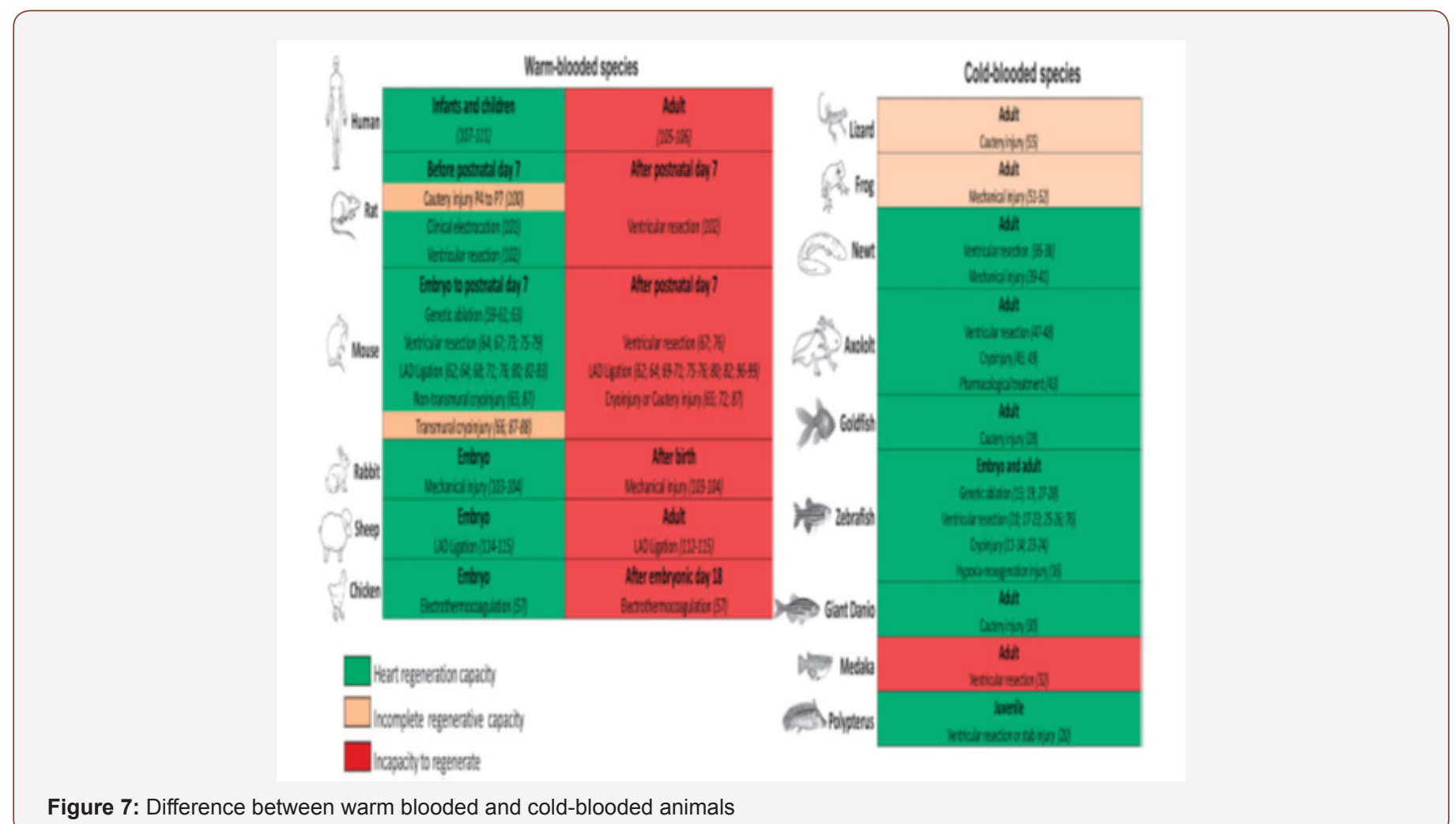

Figure 7: Difference between warm blooded and cold-blooded animals

Acute inflammation occurs after myocardial injury in regenerative and non-regenerative models. Activation of the innate immune system is one of the first events to occur after heart damage and is required for removal of dead cells and debris in the damaged tissue. Necrotic cells and debris are removed by neutrophils and macrophages at the wound site and these immune cells also release cytokines that initiate a cascade of events culminating in deposition of ECM proteins and connective scar tissue. Inflammation is considered to have a negative impact on heart regeneration by promoting fibrotic scar formation in adults. regenerating hearts can also react to injury by mounting an inflammatory response and depositing ECM, resulting in the formation of a transient fibrotic scar. Moreover, recent studies show a positive role for inflammation in regeneration and repair. For example, the activation of immune signals is known to facilitate skeletal muscle regeneration after injury, and, in the neonatal heart, acute cardiac inflammation is known to be important for the stimulation of angiogenesis and cardio-myocyte proliferation. The inflammatory factors that 
distinguish the neonatal regenerative response from the adult reparative response remain poorly defined but several recent studies point to potentially important roles for interleukin-6 and interleukin-13 signalling. Early inflammatory and -fibrotic responses in the damaged myocardium per se do not hamper heart regeneration and further studies are required to fully elucidate how tissue inflammation can direct either permanent scar formation or the heart regeneration [4] (Figure 7).

Front Immunol. 2014 Sep 23; 5: 459. Evolution of Innate Immunity: Clues from Invertebrates via Fish to Mammals. Buchmann K: "Host responses against invading pathogens are basic physiological reactions of all living organisms. Since the appearance of the first eukaryotic cells, a series of defense mechanisms have evolved in order to secure cellular integrity, homeostasis, and survival of the host. Invertebrates, ranging from protozoans to metazoans, possess cellular receptors, which bind to foreign elements and differentiate self from non-self. This ability is in multicellular animals associated with presence of phagocytes, bearing different names (amebocytes, hemocytes, coelomocytes) in various groups including animal sponges, worms, cnidarians, mollusks, crustaceans, chelicerates, insects, and echinoderms (sea stars and urchins). Basically, these cells have a macrophage-like appearance and function and the repair and/or fight functions associated with these cells are prominent even at the earliest evolutionary stage. The cells possess pathogen recognition receptors recognizing pathogen-associated molecular patterns, which are well-conserved molecular structures expressed by various pathogens (virus, bacteria, fungi, protozoans, helminths). Scavenger receptors, Toll-like receptors, and Nod-like receptors (NLRs) are prominent representatives within this group of host receptors. Following receptor-ligand binding, signal transduction initiates a complex cascade of cellular reactions, which lead to production of one or more of a wide array of effector molecules. Cytokines take part in this orchestration of responses even in lower invertebrates, which eventually may result in elimination or inactivation of the intruder. Important innate effector molecules are oxygen and nitrogen species, antimicrobial peptides, lectins, fibrinogen-related peptides, leucine rich repeats (LRRs), pentraxins, and complement-related proteins. Echinoderms represent the most developed invertebrates and the bridge leading to the primitive chordates, cephalochordates, and urochordates, in which many autologous genes and functions from their ancestors can be found. They exhibit numerous variants of innate recognition and effector molecules, which allow fast and innate responses toward diverse pathogens despite lack of adaptive responses. The primitive vertebrates (agnathans also termed jawless fish) were the first to supplement innate responses with adaptive elements. Thus hagfish and lampreys use LRRs as variable lymphocyte receptors, whereas higher vertebrates [cartilaginous and bony fishes (jawed fish), amphibians, reptiles, birds, and mammals] developed the major histocompatibility complex, T-cell receptors, and B-cell receptors (immunoglobulins) as additional adaptive weaponry to assist innate responses. Extensive cytokine networks are recognized in fish, but related signal molecules can be traced among invertebrates. The high specificity, antibody maturation, immunological memory, and secondary responses of adaptive immunity were so successful that it allowed higher vertebrates to reduce the number of variants of the innate molecules originating from both invertebrates and lower vertebrates. Nonetheless, vertebrates combine the 2 arms in an intricate inter-dependent network. Organisms at all developmental stages have, in order to survive, applied available genes and functions of which some may have been lost or may have changed function through evolution. The molecular mechanisms involved in evolution of immune molecules, might apart from simple base substitutions be as diverse as gene duplication, deletions, alternative splicing, gene recombination, domain shuffling, retrotransposition, and gene conversion. Further, variable regulation of gene expression may have played a role" [5].

Graeme J. Roch et al: “Gonadotropin-releasing hormone (GnRH) is a critical reproductive regulator in vertebrates. Homologous peptides are also found in invertebrates, with a variety of characterized functions. In the amphioxus, an invertebrate that provides the best model for the transition to vertebrates, 4 GnRH receptors (GnRHRs) were previously described, but their native ligands were not identified. Using a more sensitive search methodology with hidden Markov models, we identified the first GnRH-like peptide confirmed in the amphioxus Branchiostoma floridae. This peptide specifically activated one of the 4 GnRHRs. Although the primary structure of this peptide was divergent from any previously isolated GnRH peptide, the minimal conserved residues found in all other GnRH superfamily members were retained. The peptide was immune localized in proximity of the central canal of the anterior nerve cord, a region where other neuropeptides and receptors have been found. Additionally, the amphioxus GnRH-like gene was positioned in a locus surrounded by syntenic homologs of the human GnRH paralogon. The amphioxus GnRH-like peptide, with its distinct primary structure, activated a receptor with equal potency to multiple ligands that span the GnRH superfamily. Vertebrates and amphioxi share a number of significant characteristics including a dorsal tubular central nervous system with an anterior brain and extended spinal cord Although the other group of invertebrate chordates, tunicates, are now considered to be the closest living relatives to the vertebrates based on phylogenomic analyses amphioxi form a sister group with the rest of the chordates and in many ways provide a better model for the comparison of invertebrate protochordates with vertebrates. Several features of amphioxus including the body plan, arrangement of hox genes, synteny of neighboring genes and embryonic development are more similar to the vertebrates than those same characteristics in tunicates.

Examination of the amphioxus (Branchiostoma floridae) genome indicated that the complement of hormones and receptors controlling reproduction shared a number of similarities with the vertebrate pattern For neuroendocrine control of reproduction, kisspeptin receptor (GPR54) homologs) and kisspeptin-like sequences have been identified from the amphioxus genome, their function remains unknown In vertebrates, the kisspeptin system is 
expressed in the brain and regulates the release of gonadotropinreleasing hormone (GnRH). More recently, proof that amphioxus also expresses GnRH-like receptors was provided by cloning and in vitro activation of receptors using vertebrate $\mathrm{GnRH}$ peptides, as the endogenous amphioxus GnRH ligand(s) remained elusive. In vertebrate reproduction, the 2 pituitary gonadotropin hormones, known as luteinizing hormone ( $\mathrm{LH})$ and follicle stimulating hormone (FSH), are stimulated by GnRH. Amphioxus and tunicates lack genes encoding any of the six common vertebrate pituitary hormones, including the gonadotropins or their receptors Both protochordate groups possess genes encoding the thyrostimulin subunits which duplicated within the vertebrates to produce the glycoprotein hormone subunits of LH, FSH, and the related thyroid stimulating hormone LH and FSH act on their receptors in the gonads to stimulate gametogenesis and sex steroid synthesis. The amphioxus genome encodes 2 nuclear receptors that are similar to the sex steroid receptors in vertebrates and a set of enzymes that act in the synthesis of estradiol, testosterone, and progesterone. Although these steroids have been identified in the amphioxus gonad only estradiol has been shown to bind one of the steroidal receptors Nonetheless, amphioxus has considerable potential for reproductive cycle feedback at the level of the nervous system, gonads, and other tissues that may be targeted by the sex steroids.

The transition from invertebrate to vertebrate is hypothesized to have involved 2 rounds of whole-genome duplication in ancestral vertebrates before the radiation of lineages leading to the extant vertebrate classes The resulting genomes contained up to 4 paralogous genes, although many losses occurred. The proposed 4 copies of the original vertebrate gnrh genes have been traced by identifying their gene neighbors in syntenic analyses. GnRH4 has not been identified in extant vertebrates and is thought to have been lost early in evolution of the. Genomic analysis suggests GnRH3 was lost from the tetrapod lineage but is present throughout the teleost fish and may be homologous with lamprey GnRH-I and GnRH-III GnRH2 has been identified throughout the vertebrates including humans and has retained its conserved synteny although some mammals (e.g., mouse, rat, and chimp) have lost a functional GnRH2 gene. GnRH1 is found from bony fish to humans; there are at least nine variant forms of GnRH1 that share structure and function. In the human genome, GnRH genes or their traces (ghosts) exist on 4 different chromosomes surrounded by genes with conserved synteny: GnRH1 is encoded on chromosome 8, GnRH2 on chr. 20, a lost GnRH3 (ghost) on chr. 10, and a GnRH4 ghost on chr. 5. These conserved GnRH paralogons belong to a shared chordate ancestral linkage group (ALG 7), which was defined by comparison with homologous genes found in the amphioxus B. floridae.

The GnRH family of peptides and receptors has been proposed to be part of a larger superfamily including invertebrate-specific members. Initially, 2 invertebrate (Drosophila) receptors were shown to be structurally related to GnRH receptors (GnRHRs), but their ligands were identified as corazonin peptide and adipokinetic hormone (AKH). Further analysis of the receptor families revealed that $\mathrm{GnRH}$, corazonin, and AKH receptors were also closely related to the oxytocin, vasopressin, and crustacean cardio-active peptide (CCAP) receptors forming 2 large superfamilies with a shared ancestry that emerged before the origin of the bilaterians. In this study, evidence is presented that an amphioxus GnRH-like peptide has been identified. The cloned amphioxus gnrh-like cDNA is characterized, and the putative peptide is aligned with vertebrate GnRHs and closely related invertebrate GnRH-like peptides, corazonins, and AKHs. The bioactivity of the new peptide is tested in vitro for activity with the 4 known amphioxus GnRHRs. Synteny analysis is used to determine the lineage of this ancestral-type peptide and its relationship to vertebrate GnRHs. Phylogenetic analysis of the amphioxus receptors demonstrates a long standing and complex relationship among the receptors of $\mathrm{GnRH}$, corazonin, $\mathrm{AKH}$, oxytocin/vasopressin, and neuropeptide S" [6].

Guillaume Rivière: "DNA methylation is evolutionarily conserved. Vertebrates exhibit high, widespread DNA methylation whereas invertebrate genomes are less methylated, predominantly within gene bodies. DNA methylation in invertebrates is associated with transcription level, alternative splicing, and genome evolution, but functional outcomes of DNA methylation remain poorly described in lophotrochozoans. Recent genome-wide approaches improve understanding in distant taxa such as molluscs, where the phylogenetic position, and life traits of Crassostrea gigas make this bivalve an ideal model to study the physiological and evolutionary implications of DNA methylation. We review the literature about DNA methylation in invertebrates and focus on DNA methylation features in the oyster. Indeed, though our MeDIP-seq results confirm predominant intragenic methylation, the profiles depend on the oyster's developmental and reproductive stage. We discuss the perspective that oyster DNA methylation could be biased toward the $5^{\prime}$-end of some genes, depending on physiological status, suggesting important functional outcomes of putative promoter methylation from cell differentiation during early development to sustained adaptation of the species to the environment.

DNA methylation is an epigenetic mechanism of great biological significance which is widely conserved in evolution. DNA methylation in prokaryotes mostly affects adenines and is implicated in a broad range of processes from the control of DNA replication to the defense against bacteriophages. Besides, bacterial DNA methylation contributes to the inheritable control of gene expression, maintaining phenotypes through cell generations, as observed for virulence genes. DNA in eukaryote genomes is predominantly methylated on cytosines significant differences exist between kingdoms. In animal genomes cytosines are methylated mostly within CpG dinucleotides, whereas plants, and fungy can also have methylcytosines in the CHG or CHH contexts. In addition, genomes display markedly different methylation profiles between vertebrates and invertebrates. Vertebrates exhibit a high DNA methylation, whereas invertebrate genomes are far less methylated. In vertebrates, DNA methylation affects the whole genome, but eventually drops in the $5^{\prime}$ regions of genes which divide into 2 groups regarding their $\mathrm{CpG}$ content. The low$\mathrm{CpG}$ promoters are hypermethylated and drive the transcription 
of tissue-specific genes. Instead, the high-CpG promoters where CG dinucleotide-rich regions define "CpG islands" (CGIs) are hypomethylated and control widely expressed genes. Depending on their density, methylcytosines in promoters restrict the access of the transcription machinery to transcription start sites due to CpG-binding proteins recruitment and/or the regulation of DNA/ histone interactions within nucleosomes thereby diminishing transcription. Invertebrates generally display a heterogenic or "mosaic" methylation profile with predominant methylation of transcription units.High-throughput sequencing technologies have rapidly increased our knowledge on DNA methylation in ecdysozoans (encompassing nematodes and arthropods), especially insects.bringing new insights into the evolution of DNA methylation functions. Now, these questions have to be addressed in more distant invertebrate taxa such as lophotrochozoans (encompassing worms and molluscs), which remain widely underdescribed. Indeed, despite methylated DNA being present in the snail Biomphalaria glabrata, the scallops Chlamys farreri and Patinopecten yessoensis, the truncated wedgeshell Donax trunculus and the pacific oyster Crassostrea gigas only one single-base resolution methylome is available to date in a mollusc, the pacific oyster Crassostrea gigas. C. gigas represents an interesting species for the study of DNA methylation in lophotrochozoans, with regards to its peculiar life traits, economic and ecological importance. Indeed, C. gigas, undergoes a pelagic development and metamorphoses before a benthic adult phase in the highly stressful intertidal area. Besides, oysters are successive hermaphrodites due to a yearly gonad renewal from stem cells. the entire life cycle of oysters is punctuated with dramatic morpho-physiological changes, which rely on the implementation of transient transcriptomes within changing environments. The control of these transcriptomes likely implicates epigenetic mechanisms, which remain to be elucidated. The recent characterization of the $\mathrm{C}$. gigas genome enabled an assessment that DNA methylation was mostly intragenic in the oyster.our recent results indicate that oyster methylation patterns display temporal variations and could be uniquely biased toward the $5^{\prime}$-upstream region of gene subsets depending on physiological contexts. in this work we first review the literature on invertebrate DNA methylation focusing on gene body methylation (GBM) and highlight the current knowledge in the oyster. Then, we discuss the perspective of functional outcomes of possible specific DNA methylation features in C. gigas" [7].

Adam Siepel et al: "We have conducted a comprehensive search for conserved elements in vertebrate genomes, using genome-wide multiple alignments of 5 vertebrate species (human, mouse, rat, chicken, and Fugu rubripes). Parallel searches have been performed with multiple alignments of 4 insect species ( 3 species of Drosophila and Anopheles gambiae), 2 species of Caenorhabditis, and seven species of Saccharomyces. Conserved elements were identified with a computer program called phastCons, which is based on a 2-state phylogenetic hidden Markov model (phylo-HMM). PhastCons works by fitting a phylo-HMM to the data by maximum likelihood, subject to constraints designed to calibrate the model across species groups, and then predicting conserved elements based on this model. The predicted elements cover roughly 3\%-8\% of the human genome (depending on the details of the calibration procedure) and substantially higher fractions of the more compact Drosophila melanogaster (37\%-53\%), Caenorhabditis elegans (18\%-37\%), and Saccharaomyces cerevisiae (47\%-68\%) genomes. From yeasts to vertebrates, in order of increasing genome size and general biological complexity, increasing fractions of conserved bases are found to lie outside of the exons of known protein-coding genes. In all groups, the most highly conserved elements (HCEs), by log-odds score, are hundreds or thousands of bases long. These elements share certain properties with ultraconserved elements, but they tend to be longer and less perfectly conserved, and they overlap genes of somewhat different functional categories. In vertebrates, HCEs are associated with the $3^{\prime}$ UTRs of regulatory genes, stable gene deserts, and megabase-sized regions rich in moderately conserved noncoding sequences. Noncoding HCEs also show strong statistical evidence of an enrichment for RNA secondary structure [8]."

Remo Sanges et al: "Co-option of cis-regulatory modules has been suggested as a mechanism for the evolution of expression sites during development. the extent and mechanisms involved in mobilization of cis-regulatory modules remains elusive. To trace the history of non-coding elements, which may represent candidate ancestral cis-regulatory modules affirmed during chordate evolution, we have searched for conserved elements in tunicate and vertebrate (Olfactores) genomes. We identified, for the first time, 183 non-coding sequences that are highly conserved between the 2 groups. Our results show that all but one element are conserved in non-syntenic regions between vertebrate and tunicate genomes, while being syntenic among vertebrates. Nevertheless, in all the groups, they are significantly associated with transcription factors showing specific functions fundamental to animal development, such as multicellular organism development and sequencespecific DNA binding. The majority of these regions map onto ultraconserved elements and we demonstrate that they can act as functional enhancers within the organism of origin, as well as in cross-transgenesis experiments, and that they are transcribed in extant species of Olfactores. We refer to the elements as 'Olfactores conserved non-coding elements' [9]."

Heinrich Reichert: "Comparative studies of brain development in vertebrate and invertebrate model systems demonstrate remarkable similarities in expression and action of developmental control genes during embryonic patterning, neural proliferation and circuit formation in the brain. Thus, comparable sets of developmental control genes are involved in specifying the early brain primordium as well as in regionalized patterning along its anteroposterior and dorsoventral axes. similar cellular and molecular mechanisms underlie the formation and proliferation of neural stem cell-like progenitors that generate the neurons in the central nervous systems. neural identity and some complex circuit interconnections in specific brain domains appear to be comparable in vertebrates and invertebrates and may depend on similar developmental control genes. Despite the extensive 
history of investigations on the brain, which reaches back over 2 millenia, fundamental insight into the nature and origin of the brain remained elusive until the nineteenth century when 2 central theories of the biological sciences were first proposed. These unifying theories were the cell theory of Schleiden and Schwann, which states that all living organisms are composed of and derived from cells, and the evolutionary theory of Darwin, which explains the origin of organismal complexity and diversity through the action of natural selection. The subsequent successful application of both theories to the analysis of vertebrate brains uncovered fundamental similarities in the neural organization of different vertebrate brains, arguing for a common evolutionary origin of major brain elements in all vertebrates. By contrast, when the nervous systems of vertebrates were compared with those of invertebrates, apparent differences in neuroanatomy were found in both adult structure and embryonic morphogenesis. Based on these differences, a number of investigators proposed an independent evolutionary origin for the nervous systems of vertebrate and invertebrate animals. Contrasting with this notion, and in support of earlier ideas are more recent neurogenetic analyses carried out in several vertebrate and invertebrate model systems that reveal striking similarities in the expression and action of regulatory genes that control brain development. Indeed, comparable developmental genetic mechanisms appear to operate in invertebrates and vertebrates during all major stages of brain development from the early specification of the neuroectoderm to the formation of neural circuitry. In the following, I consider 4 key aspects of brain development as well as the underlying molecular genetic control elements that appear to be conserved.

\section{Generation of the neuroectoderm}

At the earliest stage of brain development, a conserved molecular signalling system polarizes the embryonic ectoderm into a neuroectoderm, from which the central nervous system (CNS) develops, and into a 'non-neural' ectoderm. A key element in this signalling system is the Dpp/Bmp protein, a member of the TGFb gene family, which is thought to exert an antineurogenic effect that represses neural identity and limits the extent of the neuroectoderm to one side of the embryo. The expression of active Dpp/Bmp on this side is prevented by a second key element in the conserved signalling system, the Sog/Chordin protein, which antagonizes the action of the Dpp/Bmp morphogen. Whenever a CNS develops, in vertebrates or in invertebrates, it is always formed on the side of the body that does not express Dpp/ Bmp, be it ventral in invertebrates, or dorsal in vertebrates.

\section{Self renewal and proliferation of neural stem cells}

Following their generation, neural stem cells initiate proliferation by self-renewing and producing neural progeny. There are remarkable similarities in the action of specific developmental control genes during this process in vertebrate and invertebrate brains. the Activin, Notch, Hedgehog and Fibroblast growth factor signaling pathways are active and have comparable roles in maintaining neural stem cell self-renewal and/ or proliferation in the developing CNS of Drosophila and mouse. the molecular components that regulate the asymmetric nature of the neural stem cell divisions, including regulators of cell polarity and determinants of daughter cell fates, have highly conserved counterparts in invertebrates and mammals. In addition to these molecular biological similarities, there are striking parallels in the cell biology of neural stem cell proliferation in vertebrates and invertebrates. In the mammalian embryonic CNS, neural stem cells can self-renew while producing secondary progenitors that each divide once to form two post-mitotic neurons. This corresponds to the proliferation of insect neural stem cells ('neuroblasts') that can also self-renew while producing secondary progenitors ('ganglion mother cells') that divide once to form 2 neurons. In mammalian brain development, neural stem cells can also self-renew and generate amplifying intermediate progenitors, each of which retains some self-renewal potential and can generate multiple neural progeny. Remarkably, a similar mode of amplifying neural stem cell proliferation has recently been discovered in Drosophila. Specific brain neuroblasts in the fly brain divide asymmetrically in stem cell mode to self-renew and generate intermediate progenitors that subsequently divide multiple times in stem cell mode, thus amplifying the number of progeny produced [10]."

Shengfeng Huang et al: "Vertebrates diverged from other chordates B500 Myr ago and experienced successfulinnovations and adaptations, but the genomic basis underlying vertebrate origins are not fully understood. Here we suggest, through comparison with multiple lancelet (amphioxus) genomes, that ancient vertebrates experienced high rates of protein evolution, genome rearrangement and domain shuffling and that these rates greatly slowed down after the divergence of jawed and jawless vertebrates. Compared with lancelets, modern vertebrates retain, at least relatively, less protein diversity, fewer nucleotide polymorphisms, domain combinations and conserved non-coding elements (CNE). Modern vertebrates also lost substantial transposable element (TE) diversity, whereas lancelets preserve high TE diversity that includes even the longsought RAG transposon. Lancelets also exhibit rapid gene turnover, pervasive transcription, fastest exon shuffling in metazoans and substantial TE methylation not observed in other invertebrates. These new lancelet genome sequences provide new insights into the chordate ancestral state and the vertebrate evolution [11]."

Philip C. J. Donoghue et al: "The evolutionary origin of vertebrates has been debated ad nauseam by anatomists, paleontologists, embryologists, and physiologists, but it is only now that molecular phylogenetics is providing a more rigorous framework for the placement of vertebrates among their invertebrate relatives that we can begin to arrive at concrete conclusions concerning the nature of ancient ancestors and the sequence in which characteristic anatomical features were acquired. Vertebrates tunicates and cephalochordates together comprise the chordate phylum, which along with echinoderms and hemichordates constitute the deuterostomes. The origin of vertebrates and of jawed vertebrates is characterized by a doubling of the vertebrate genome, leading to hypotheses that this 
genomic event drove organismal macroevolution. This perspective of evolutionary history, based on living organisms alone, is an artifact. Phylogenetic trees that integrate fossil vertebrates among their living relatives demonstrate the gradual and piecemeal assembly of the gnathostome body plan. Unfortunately, it has not been possible to demonstrate gradual assembly of the vertebrate body plan. This is not because vertebrates are irreducibly complex but because many of the characters that distinguish vertebrates from invertebrates are embryological and cellular and, inherently unfossilizable Humans and all other back-boned animals-plus a few others that have no bone at all-comprise the vertebrates.

Vertebrates are a clade, meaning that all members of the group have evolved from a common ancestor that they all share. This means that the deeper parts of our evolutionary history are entwined with the origin of the clade, and it should thus come as no surprise to discover, that the origin of vertebrates has been the subject of intense debate since the earliest days of evolutionary research. In his book Before the backbone, Henry Gee recounts a great number of theories that, over the last century and a half, have invoked almost every other major living animal group as the ancestors of vertebrates. Mercifully, there is now much less equivocation over the relationships of vertebrates to their living relatives, none of which are thought of as being ancestral. vertebrates and their nearest kin-the invertebrate chordates, the hemichordates and the echinoderms-are more correctly perceived as living representatives of distinct genealogical lineages that separated one from another deep in geological time. It is the aim of many paleontologists, comparative anatomists, embryologists, and molecular biologists to uncover the genealogical relationships of these animals-their family tree-and to test this tree with evidence that bears on the question of how these distinct organismal designs. Explanations of the emerging evolutionary pattern range from traditional Darwinian gradualistic evolution to those that invoke explosive diversifications (seized upon by creationists and intelligent designers as evidence for irreducible complexity but which are actually consistent with natural causal mechanisms Whatever the pattern and processes, a holistic understanding of the origin and diversification of vertebrates can only be obtained by a holistic approach, integrating all relevant strands of evidence into a framework of evolution-ary relationships established on the basis of the only uni-versal characteristics shared by all organisms: molecular sequence data [12] (Figure 8)."

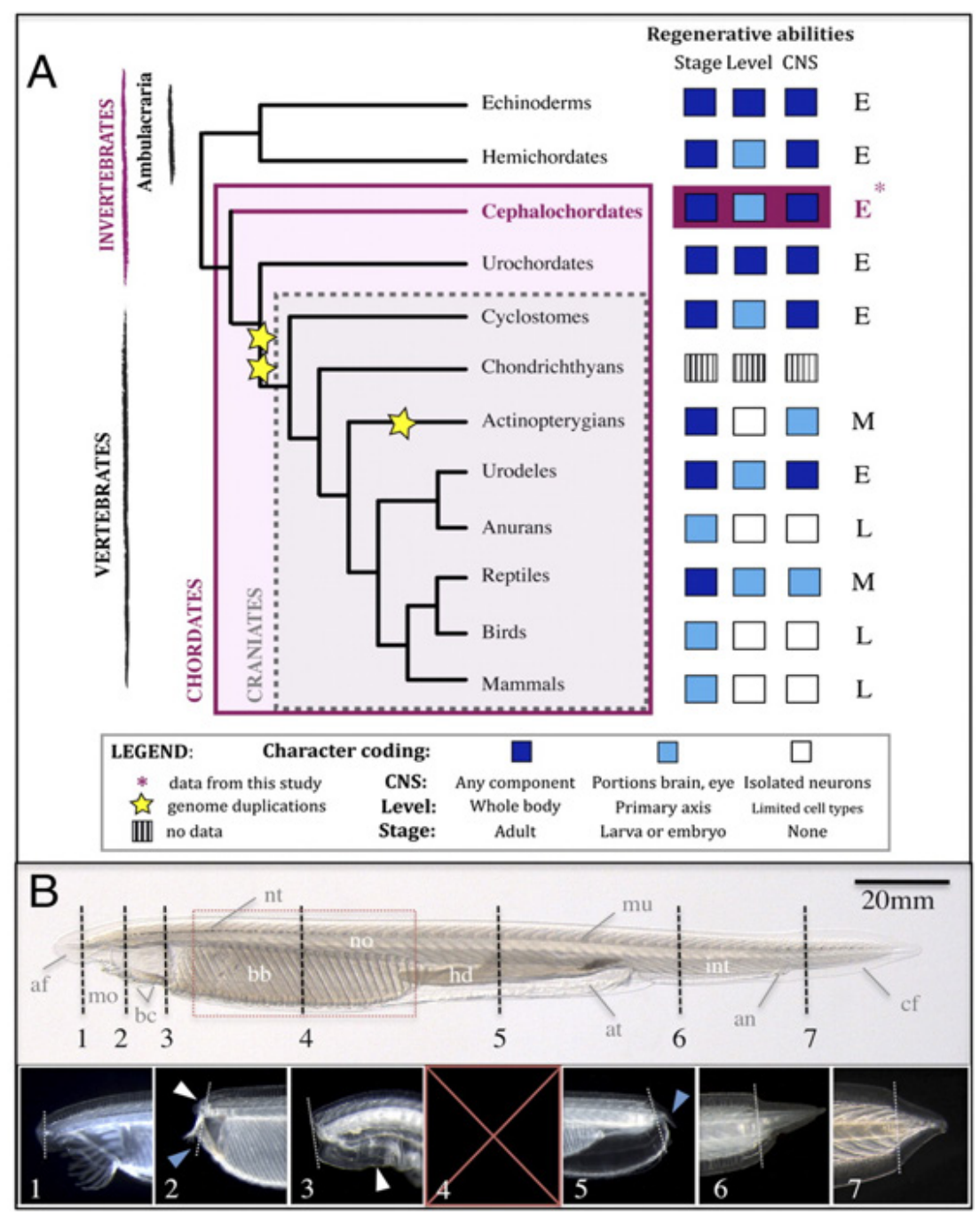

Figure 8: Regenerative abilities. 
Esther T Chan, et al: "Vertebrates share the same general body plan and organs, possess related sets of genes, and rely on similar physiological mechanisms, yet show great diversity in morphology, habitat and behavior. Alteration of gene regulation is thought to be a major mechanism in phenotypic variation and evolution, but relatively little is known about the broad patterns of conservation in gene expression in non-mammalian vertebrates. We measured expression of all known and predicted genes across twenty tissues in chicken, frog and pufferfish. By combining the results with human and mouse data and considering only ten common tissues, we have found evidence of conserved expression for more than a third of unique orthologous genes. We find that, on average, transcription factor gene expression is neither more nor less conserved than that of other genes. Strikingly, conservation of expression correlates poorly with the amount of conserved nonexonic sequence, even using a sequence alignment technique that accounts for noncollinearity in conserved elements. Many genes show conserved human/fish expression despite having almost no nonexonic conserved primary sequence. There are clearly strong evolutionary constraints on tissue-specific gene expression. A major challenge will be to understand the precise mechanisms by which many gene expression patterns remain similar despite extensive cis-regulatory restructuring. Vertebrates all share a body plan, gene number and gene catalog inherited from a common progenitor, but so far it has been unclear to what degree gene expression is conserved. King and Wilson initially posited that phenotypic differences among primates are mainly due to adaptive changes in gene regulation, than to changes in protein-coding sequence or function, and this idea has accumulated supporting evidence in recent years. Recent work has indicated that gene expression evolves in a fashion similar to other traits, where in the absence of selection, random mutations introduce variants within a population. Changes negatively affecting fitness are probably eliminated by purifying selection: core cellular processes seem to be coexpressed from yeast to human, and conservation of the expression of individual genes in specific tissues has been observed across distantly related vertebrates, perhaps reflecting requirements for patterning and development as well as conserved functions of organs, tissues and cell types. Conversely, changes that benefit fitness (for example, under new ecological pressures) may become fixed: changes in gene expression are believed to underlie many differences in morphology, physiology and behavior and, indeed, subtle differences in gene regulation can result in spatial and temporal alterations in transcript levels, with phenotypic consequences at the cell, tissue and organismal levels. The degree to which stabilizing selection constrains directional selection and neutral drift across the full vertebrate subphylum is, to our knowledge, unknown.

Comparative genomic analyses provide a perspective on the evolution of both cis- and trans-regulatory mechanisms, and they are often used as a starting point for the identification of regulatory mechanisms. One estimate, using collinear multiple-genome alignments, suggested that roughly a million sequence elements are conserved in vertebrates (particularly among mammals, which represent the majority of sequenced vertebrates), with most being nonexonic, and a series of studies have demonstrated the cis-regulatory potential of the most highly conserved nonexonic elements (for example,. Another study found that only $29 \%$ of nonexonic mammalian conserved bases are evident in chicken, and that nearly all aligning sequence in fish overlaps exons, raising the possibility that gene regulatory mechanisms may be very different among vertebrate clades. Absence of conserved sequence does not imply lack of regulatory conservation, as many known cisregulatory elements seem to undergo rapid turnover, and there are examples in which orthologous genes have similar expression patterns despite apparent lack of sequence conservation in regulatory regions. As further evidence of pervasive regulatory restructuring in vertebrate evolution, an analysis that accounted for shuffling (non-collinearity) of locally conserved sequences suggested that the number of conserved elements may be several fold higher than collinear alignments detect, particularly between distant vertebrate relatives, such as mammals and fish.

Trans-acting factors (transcription factors or TFs) also show examples of striking conservation, such as among the homeotic factors, and diversifying selection. Studies comparing expression patterns between human and chimpanzee liver found that TF genes were enriched among the genes with greatest humanspecific increase in expression levels, supporting arguments for alteration of trans-regulatory architecture as a driving evolutionary mechanism. On the other hand, in the Drosophila developmental transition, expression of transcription factor genes is more evolutionarily stable than expression of their targets, on average. The fact that enhancers will often function similarly in fish and mammals, even when the enhancer itself is not conserved, indicates that mechanisms underlying cell-specific and developmental expression are likely to be widely conserved across vertebrates. Global trends in conservation of gene expression, conservation of cis-regulatory sequence and relationships between the 2 are not completely understood, partly because the cis-regulatory 'lexicon' (that is, how TF binding sites combine to form enhancers) remains mostly unknown, testing individual enhancers is tedious and expensive, and many vertebrates are not amenable to genetic experimentation. These issues are of both academic and practical consequence: in addition to our curiosity about the origin and distinctive characteristics of the human species, primary sequence conservation is widely used to identify regulatory mechanisms. We reasoned that expression profiling data from species spanning much greater phylogenetic distance than humans and mice, and thus having greater opportunity for both neutral drift and positive selection, would allow assessment of the degree of conservation of tissue gene expression among all vertebrates, and a comparison of the conservation of expression to the conservation of nonexonic primary sequence. Here, we describe a survey of gene expression in adult tissues and organs in the main vertebrate clades: mammals, avians/reptiles, amphibians and fish. Our analyses demonstrate that core tissue-specific gene expression patterns are conserved across all major vertebrate lineages, but that the correspondence 
between conservation of expression and amount of conserved nonexonic sequence is weak overall, at least at a level that is detectable by current alignment approaches [13]."

Laura Pulze et al: "Telocytes, a peculiar cell type, were recently found in vertebrates. Hence this cell system has been reported as ubiquitous in the bodies of mammals and interpreted as an important player in innate immunity and tissue regeneration, it is reasonable to look for it also in invertebrates, that rely their integrity solely by innate immunity. Here we describe, at morphological and functional level, invertebrate telocytes from the body of leech Hirudo medicinalis (Annelida), suggesting how these cells, forming a resident stromal 3D network, can influence or participate in different events. These findings support the concepts that leech telocytes: i) are organized in a cellular dynamic and versatile 3D network likewise the vertebrate counterpart; ii) are an evolutionarily conserved immune-neuroendocrine system; iii) form an immuno-surveillance system of resident cells responding faster than migrating immunocytes recruited in stimulated area; iv) communicate with neighbouring cells directly and indirectly, via cell-cell contacts and soluble molecules secreted by multi vesicular bodies; v) present within neo-vessels, share with immunocytes the mesodermal lineage; vi) are involved in regenerative processes. we propose that HmTCs, integrating so different functions, might explain the innate immune memory and can be associated with several aged related diseases. The present paper provides evidence for the presence of TCs in the leech H. medicinalis which in this context is considered a good invertebrate animal model. This organism shows a "parenchimatous" body in which the loose connective tissue is colonized by several and different types of stromal cells such as macrophages, fibroblasts, myo-fibroblasts, NK-like cells and granulocytes, that share the same phenotype and function closely to the ver-tebrate counterpart. In addition, the basic steps of cellular responses, evoked by different stimuli, are strikingly similar to those observed in higher forms of life. Among the leech stromal cells, the HmTCs can be detected due to their affinity for specific vital dyes. In non-stimulated animals, HmTCs in resting condition show the typical morphology described for vertebrate TCs: a pyriform or small ovoidal cell body with very long and thin cytoplasmic processes elongated up to $50 \mu \mathrm{m}$ that are occupied by mitochondria, bunches of filaments perpendicularly oriented and few granules. The HmTC branches extend them in different directions and are engaged in cell-cell and cell-matrix contacts. The HmTCs form a network interconnecting cells from muscle layers, vessels, nervous system as well as collagenous bundles of connective tissue filling the leech body. Once activated (after injection of LPS or following surgical lesion), these cells, organized in a plastic and dynamic system, can change their phenotype, acquiring an elongated shape suitable for migration with shorter telopods characterized by the involvement of the cytoskeletal elements. HmTCs in healthy and in activated leeches are identified, as in vertebrate (in human) by double-positive immune staining with CD34/vimentin, c-kit/vimentin, c-kit/CD34 and c-kit/Oct-4 which are stem cell markers expressed simultaneously with the hallmarks of TCs.It is important to underline that another important characteristic of HmTCs is that they can recognize micro-bial components through TLRs that mediate activation of innate immunity. The detection of the non-self results in the inflammation phase with cell surface activation molecules followed by secretion of pro-inflammatory cytokines such as IL-18. These multifunctional cells, as several innate immune cells, express also HmAif-1, other factor typically involved in inflammatory responses. HmTCs, similarly to the major inflammatory cell types (immunocytes), are equipped to produce hormones such as ACTH and are able to respond to them. In our previous studies we have demonstrated that ACTH loop (controlling locally immune reactivity and regulating the cellular functions of the immune system in an autocrine and paracrine manner) has a key role in mutually influencing immune and neuroendocrine functions. The HmTC complex, that we defined as a "system in the system", represents the most primitive immune organ-ization, participating and conducting the first line of tissue/organism defences. The powerful ability of HmTC stromal network is due to the 3D organization that guarantees cross-talk among various cell types via intercellular contacts, secretion of cytokines and release of extracellular vesicles and exosomes.These cells share with the various types of immune cytes the same mesodermal lineage as evidenced by their presence, after stimulation, inside the lumen of neo-vessels where are strictly connected with leukocyte-like cells. Experiments with the vital marker Dil-Ac-LDL, which is taken up preferentially by endothelial cells and pre-cursor cells, are useful for staining and following the fate of these cellular types and to confirm the presence of HmTCs localized among precursors of circulating cells, within the lumen of newly formed vessels and dispersed among the groups of muscle cells or localized close to the injured region in wounded leeches. This finding rein-forced the concept that hematopoietic precursor cells and telocytes, both of which develop in close association, have a mesenchymal common origin as in vertebrates. Since HmTCs configure a 3D network of resident sentinels embedded in the connective tissue matrix, they can detect the first sign of non-self presence and are required for proper communication among different cell types in all regions of the body. The HmTC immuno-surveillance system, obtained by direct cell-cell contacts and by soluble mediators released in loco, controls and directs the activity of neighboring body cells. Once activated, the first response of HmTCs results in an alert centripetally and centrifugally directed. These communication precede the responses of integrated systems (cellular and soluble molecules) that the organism utilizes against any type of non-self. HmTC crosstalk by-passes both the slowness of the other types of innate immune cells that have to migrate and increase numerically before becoming operative and the slower spread, via matrix, of soluble molecules (growth factors and cytokines) that are produced by those cells directly involved in lesion or injected sites. Thanks to the characteristics of stem cell, HmTCs are also engaged in regenerative processes at the lesion area. Overall detailed analysis demonstrates that HmTCs are organized in a primitive ancient 3D system, part of innate immunity both in invertebrates and vertebrates. The fact 
that invertebrates and vertebrates could share cells, molecules and related functions inserted on a basic frame is not surprising according to an economic, con-servative strategy in which a basic cellular network can provide the first line of body/tissue defence followed by more specific responses. due to the features of HmTC network we extend the list of leech defense mechanisms by addressing the problem of immunological memory in invertebrates. We have already suggested the existence of a kind of positive immune memory in allo- and xeno-transplantation in leeches 40 and demonstrated that in second-set grafting experiments, responses to the second transplant were always faster and stronger than those occurring in first-set grafting experiments. Now we hypothesize that this form of innate immune memory (trained immunity) in invertebrate, unrelated to antibodies that mediate adaptive immunity, could be explained by how with the HmTCs' function.Indeed, a provocative thought arises from the intriguing comparison of HmTC network with microglia cells, not only in leeches but also in vertebrates. Interestingly microglia and TCs can be considered as the ancient conserved interface-system between immune and neuroendocrine function because share similar mediators and receptors from lower invertebrate up to human61-67. Support for this idea comes from comparative studies show-ing that TCs and microglia from $\mathrm{H}$. medicinalis and vertebrates present a number of similarities in morphol-ogy, cellular functions, cell surface antigens, histochemical properties, mesodermal origin, capability to migrate, proliferate and differentiate in response to injury. It could be of interest to evaluate the possibility that TCs and microglial cells could represent the same type of cell resident in different districts able to detect the first signs of non-self invasion or tissue damage [14]".

Zhuo Wang et al: "Heart disease remains the leading cause of death world-wide and its prevalence is likely to increase further withchanges in lifestyle and as the population ages. there is an urgent need for new drugs that are effective intreating heart disease patients. Cardiacfibrosis is implicit inall forms of heart disease. Fibrosis is a scarring process characterized by myofibroblast accumulation and excessive deposition of extracellular matrix (ECM) proteins, in particular collagen I and III. This can lead to loss of organ architecture and compliance, induction of pathological signaling in cardio myocytes and eventual heart failure. The fibrotic process is similar in many organs, including lung, liver and kidneys, making it an attractive target for therapeutic intervention. targeted therapy is complicated, as the fibrosis causing myofibroblasts can originate from multiple cell types including endothelial cells (ECs) (known as endothelial-mesenchymal transition, EndMT)1, pericytes2, epithelial cells (epithelial-mesenchymal transition, EMT)3 and fibroblasts 4. During cardiac fibrosis, genetic lineage studies indicate that the majority of myofibroblasts result from resident cardio fibroblasts. End MT-derived myofibroblasts may also play an importantrole1,6, particularly in the loss of resident blood vessels, in the area of tissue damage via capillary rarefaction. The transforming growth factor $\beta$ (TGF $\beta$ ) family of growth factors are pivotal in driving the transition of fibroblasts, endothelial cells, pericytes and epithelial cells into active myofibroblast in response to fibrotic stimuli8-10.The most documented member associated with fibros is development is TGF $\beta 1$. Mature TGF $\beta 1$ ispartofalatentcomplex consisting of a TGF $\beta 1$ dimer noncovalently bound to its latency-associated peptide (LAP), which is associated to a large TGF $\beta$-binding protein (LTBP). Once activated, TGF $\beta 1$ binds to its ubiquitously expressed cell surface TGF $\beta 1$ type I and type II receptors, leading to the activation of a downstream signalling cascade involving both canonical, e.g., phosphorylation of Smad proteins and non-canonical signalling. This leads to the transcriptional regulation of a range of genes involved in the transition of cells into myofibroblasts leading to increased matrix deposition and fibrosis. Transglutaminase 2 (TG2) is a multifunctionalCa2+-dependent protein crosslinking enzyme, which is regulated by TGF $\beta 1$ and also involved in the activation of matrixbound latent TGF $\beta 11$. Proof-of-concept studies using animal models indicate TG2 is involved in lung12andkidneyfibrosis13, where it has a role in matrix deposition and accumulation, and in latent TGF $\beta 1$ activation. How-ever, transglutaminase inhibitors were only used in kidney fibrosis models and the inhibitors used in these studies were not selective for TG213. We have now developed via the aid of in silico modelling highly potent TG2 selective inhibitors capable of reducing angiotensin II (AngII)-induced nephron sclerosis in mice 14 . We demonstrated a mechanism for the highly potent cell permeable compound 1-155whereby the inhibitor reduced both TG2 activity and export of TG2 into the ECM by blocking its cell surface interaction with its binding partner syndecan-413, which is required forTG2 secretion15.Given our earlier preliminary data suggesting the importance of TG2 in kidneyfibrosis14, we sought to clarify its role in the development of cardiac fibrosis using for the first time an inhibitor that is selective for TG2. Our data obtained from both cell models and 2 well-characterized in vivo models indicate a role for TG2 in the development of cardiac fibrosis. We demonstrate that theTG2 selective inhibitor 1-155 reduces fibrosis in vitro and in 2 clinically relevant mouse models of cardiac fibrosis, making TG2 an attractive drug target for anti-fibrotict herapies [15]."

Marina Pozzolini et al: "Exposure to crystalline silica particles causes silicosis, an occupational disease leading to an overproduction of collagen in the lung. The first step of this pathology is characterized by the release of inflammatory mediators. Tumor necrosis factor (TNF) is a pro-inflammatory cytokine directly involved in silica-induced pulmonary fibrosis. The marine demosponge Chondrosia reniformis is able to incorporate silica grains and partially dissolve the crystalline forms apparently without toxic effects. In the present work C. reniformis tissue explants were treated with fine quartz dust and the expression level of fibrogenic genes was assayed by qPCR, demonstrating an overexpression of a fibrillar and a non-fibrillar collagen and of prolyl4-hydroxylase enzyme. The deposition of new collagen could also be documented in quartz-treated sponge explants. Furthermore, TNF pro-inflammatory cytokine over expression and involvement in silica-induced sponge collagen biosynthesis was demonstrated 
in quartz-treated explants as compared with controls by means of specific TNF inhibitors affecting the fibrogenic gene response. As no documentable detrimental effect was observed in treated explants, we conclude that the C. reniformis unique quartz engulfment and erosion is physiological and beneficial to the animal, leading to new collagen synthesis and strengthening of the body stiffness. Thus, we put forward the hypothesis that an ancient physiological behaviour from the lowest of the Metazoa, persisting through evolution via the same molecular mediators such as TNF, may have become the cause of disease in the specialized tissues of higher animals such as mammals [16]."

Susan E Wilson-Sanders: "Invertebrate animals have been used as medicinals for 4,000 years and have served as models for research and teaching since the late 1800s. Interest in invertebrate models has in-creased over the past several decades as the research com-munity has responded to public concerns about the use of vertebrate animals in research. As a result, invertebrates are being evaluated and recognized as models for many diseases and conditions. Their use has led to discoveries in almost every area of biology and medicine-from embryonic development to aging processes. Species range from terrestrial invertebrates such as nematodes and insects to freshwater and marine life including planarians, crustaceans, molluscs, and many others. The most often used models are the fruit fly Drosophila melanogaster and the minuscule nematode Caenorhabditis elegans. Topics in this article are categorized by biologic system, process, or disease with discussion of associated invertebrate models. Sections on bioactive pro ducts discovered from invertebrates follow the models section, and the article concludes with uses of invertebrates in teaching. The models reviewed can serve as references for scientists, researchers, veterinarians, institutional animal care and use committees (IACUCs), and others interested in alternatives to vertebrate animals Macrophages Invertebrates have played a key role in the history of the macrophage. The term "macrophage" (phagocyte) was coined by Ilya Ilyich Mechnikov, a comparative embryologist and winner of the Nobel Prize in 1908. He observed in starfish larvae a group of cells that had unusual characteristics-the ability to move in tissue: after introducing small rose thorns into the larvae, he noted the next morning that the thorns were surrounded by the mobile cells. He further studied this phenomenon using the freshwater $\mathrm{fl}$ ea (Daphnia magna), exposing itto fungal spores: the spores were attacked and isolated by the Daphnia macrophages. Macrophagelike cells are present in many species of invertebrates. Often they originate from mesenchymal, endothelial, or fi broblastic cells that differentiate into phagocytes For example, Hydra, a member of the phylum Cnidaria, has cells with phagocytic capability that play a role in the animal's ability to recognize "self" and, thus, make it a useful model of graft rejection. Molluscs also have cells that can act as macrophages, but the origin of these cells is mesenchymal, not hematopoietic, indicating divergent paths of differentiation [17]."

Sajal Ray et al: "Invertebrates constitute more than $95 \%$ of the existing biodiversity of the world. They present an extreme range of variation in terms of their body architecture, adaptation, food preference, habitat preference, behaviour and physiological response against environmental stressors. Evolutionarily, they are an ancient group of animals which have survived an extreme range of environmental adversities and perturbations from the time of their origin. Invertebrates occupied diverse kinds of habitats including terrestrial, freshwater, marine and estuarine ecosystems. Aquatic invertebrates are usually distributed in the multiple spatial compartments of the water bodies from the surface to the bottom region called the benthic stratum. Aquatic invertebrates are also found in the conspicuous ecological areas of the mud-water interface and subsoil region at the floor of aquifers. Freshwater ponds of India serve as habitat for diverse aquatic organisms including members of Porifera, Mollusca and Arthropoda. Majority of the ecotoxicological research was carried out on Eunapius carteri (Porifera: Demospongiae: Spongillidae); Bellamya bengalensis (Mollusca: Gastropoda: Prosobranchia); Pila globosa (Mollusca: Gastropoda: Ampullariidae) and Lamellidens marginalis (Mollusca: Bivalvia: Eulamellibranchiata). Aquatic invertebrates bear ecological, economical, nutritional and biotechnological significance and demand a special scientific attention. Many of them are the dietary source of nutrition for human and other organisms. Their importance as potential source of bioactive substances and pharmacologically active compounds cannot be denied. Current scientific reports indicate that the rapid and unrestricted contamination of freshwater and marine ecosystem by diverse pollutants poses serious ecotoxicological threat for the existence of invertebrates in their natural habitats. Various anthropogenic activities like habitat destruction and habitat contamination have been identified as the causative factors of dwindling of

invertebrate species on earth. Continuous addition of various toxic chemical compounds into the global environment is a major environmental challenge encountered by human and other organisms. Many of these environmental pollutants are of either industrial or agricultural origin and need a thorough toxicological screening in invertebrate models. The overall evolutionary success and survival efficacy of invertebrates depended on several factors including the development of a highly advanced immunological system. Immunology deals with the typical strategies of biological defence against environmental toxins and pathogens. Components of immune system and their coherent functional attributes enabled the invertebrates to overcome the toxin-induced chemical stresses of the primitive and modern global environment. Many of the environmental pollutants of the current hydrosphere are less researched, with limited toxicological information. Invertebrates over a period of time are assumed to evolve novel and unique modes of immunological reactivities to defend against the toxic insults of environmental pollutants at the cellular and molecular levels. Aquatic invertebrates are relatively a neglected group of organisms with reference to their immunotoxicological status in a biounsafe environment. The principal immunotoxicological responsiveness of invertebrates centre around the reactivities of circulating immunocytes and selected effector organs like gill, digestive gland, intestine, labial palp, etc [18]." 
G De Vico et al: "We read with interest the recent paper by Tascedda and Ottaviani (2014) about the occurrence of tumors in invertebrates. In order to further reinforce your statements that "histological and molecular biology studies have proved the existence of tumors in invertebrates" we would like to add to some insights focusing on molluscan neoplasia, an emerging animal model for human cancer. It is known that neoplasia is a pathological process characterized by an overgrowth of a new tissue in the context of a pre-existing one, and consists of atypical cells, a term which incorporates the sum of the differences in morphological, biochemical and functional features of cancer cells relative to normal cells. neoplastic tissue is characterized by a self-growing, progressive, irreversible and non-finalistic behavior.2 predominant types of neoplasia have been reported in marine molluscs, viz. disseminated neoplasia, also called leukaemia or hemic neoplasia (HN), andgonadal neoplasia. In HN neoplastic cells are represented by atypical hemocytes, which display high nucleus to cytoplasm ratios, diffuse chromatin patterns and pleomorphic nuclei, and usually infiltrate tissues and organs of affected individuals.Since the initial description of the disease its cause has not been clearly defined. Viral infection, genetic profile, environmental changes and anthropogenic pollution have been proposed as the causative factors. The prevalence of the disease ranges from 0.5 to $73.3 \%$, according to the species considered and geographical origins of molluscs. In the affected bivalves HN have been for a long time considered a phenotypically similar proliferative disease in the different shellfish species involved. differencesin neoplastic cell morphology, along with descriptions of neoplastic hemocyte subtypes, have frequently contradicted this assumption Recently, we described at last 2 different types of leukemia in 2 different bivalve species (Mytilus galloprovincialis and Cerastodema edule), showing distinctive morphological and histo-pathogenetic behaviour of cells.In particular, in mussels, 2 predominant types of neoplastic cells (A and B) have been described, differently to common cockle where one population of cells have been observed; atypical cells in the respective species also showed a distinct pattern of PCNA (Proliferating Cell Nucler Antigen) expression, nuclear or cytoplasmic. Such difference could be indicator of a different mechanism of neoplastic cells initiation/progression in early and advanced phase of the disease, respectively, as also strongly supported by Diaz et al. (2013). Gonadal neoplasia is mainly represented by germinoma, which consists of a proliferation of atypical germ cells. Germinoma has been described in several species of marine bivalve molluscs, and most consistently in some populations of Mercenaria mercenaria, Mya arenaria and razor clam, Ensis arcuatus. In the above species, the prevalence of the disease in a given population could remain underestimated particularly in early cases, where neoplastic cells may still go undetected if the tissue section examined does not happen to contain them. In fact, the probability of correctly diagnosing the presence of gonadal neoplasia in molluscs increases with disease progression. 3 evolutive stages of the disease could be observed in M. arenaria, and 4 stages in Mercenaria spp., according to the percentage of gonadal follicles involved and the extent of tissue invasion. Although the aetiology or causes of neoplasia remains unclear, pollution by carcinogenic agents is implicated in the heavily exploited littoral zones of coastal waters. Germinomahave been described also in the gastropod Patella coerulea, accompanied by other gonadal developmental disorders. Many genes and pathways critically involved in neoplastic transformation and metastasis are evolutionarily conserved in molluscs. Some molecular evidence regarding stress biology and relationships to human biology is available in relation to the function of p53 superfamily members in bivalves. In particular, literature reportsp53 (which is among the best known molecules involved in vertebrates carcinogenesis) is demonstrably involved in both bivalve HNs and germinoma Both structurally and functionally, bivalve p53 family proteins are the most highly conserved members of this gene superfamily so far identified outside of higher vertebrates and invertebrate chordates (protein sequences are 67 - $69 \%$ conserved with human p53). while in vertebrates p53, p63 and p73 originating from different genes, isoforms of p53 of bivalve molluscs are splice variants of a single gene The p53 protein was detected in tumor cells of molluscs Mytilus edulis, Mytilus trossulus, M. arenaria, Spisula solidissima, Crassostrea rhizophoae and Crassostrea gigas. In particular, a homologue of the human Hsp53 protein was cloned and characterized in M. arenaria affected by $\mathrm{HN}$ and presents a domain II-V DNA-ligand, a transactivation domain and a domain MDM2 stored for $73 \%$ compared to the human p53, suggesting that the molecular mechanisms that regulate the transcription of the p53 gene in mollusks are similar to those involved in the human gene (Kelley et al., 2001). Furthermore, in neoplastic hemocytes of M. arenaria, themortalin(a member of the Hsp70 family whose expression is strongly correlated with the levels of expression of p53 in sick bivalve) sequesters inactivated p53 in the cytoplasm. A similar phenotype, characterized by Hsp70 cytoplasmic sequestration of p53 protein, has been observed in several human cancers (undifferentiated neuroblastoma, retinoblastoma, colorectal and hepatocellular carcinomas, and glioblastoma). clam hemocyte cancer is the only animal model thus far investigated where cytoplasmically sequestered wild-type p53 can be reactivated both in vitro and in vivo using both genotoxic and non-genotoxic therapies. Results suggest that mortalin-based cytoplasmic sequestration of wild-type p53 in cancerous clam hemocytes can be reversed by treatment with antineoplastic drugs also employed against similar human diseases and will result either in transcription based apoptosis when the nucleus is accessible or non-transcription-based apoptosis when nuclear access is blocked [19]."

Keith R. Cooper: "The ability to demonstrate metastasis in an invertebrate is a difficult problem for several reasons: (1) many invertebrates have no circulatory system (2) the majority of invertebrates that do have a circulatory system is based on an open system with large blood sinuses (3) many invertebrates have non discrete organ systems (4) relatively few neoplasms have been reported in invertebrates and (5) the majority of neoplasms that have been described are anaplastic in ap pearance. Metastases 
are the occurrence of tumor implants arising independently from the primary tumor or a second $\neg$ ary metastasis. In invertebrates that do not have a circulatory system the ability of neoplastic cells to migrate to secondary sites is restricted. The majority of neoplasms that have been described in invertebrates are anaplastic in appearance and are believed to arise from hematopoietic tissues. Because of the open sinusoidal blood systems it is difficult if not impossible to distinguish areas where the neoplastic cells perculate through the tissues and metastasize [20]."

Jacques Robert: "Despite intense study in mammals, the different roles played by the immune system in detecting (immunosurveillance), controlling and remodeling (immunoediting) neoplasia, and perhaps in metastasis are not fully understood. In this review, I will present evidence of neoplasia and invasive malignancy, as well as tumor immunity in invertebrates and nonmammalian vertebrates. I will also present a comparative and evolutionary view of the complex interactions between neoplasia and the host immune system. Overall, I wish to go beyond the too simplistic dichotomy between invertebrates with innate immunity that are only affected with benign neoplasia and vertebrates with adaptive immunity that are affected by metastatic malignancies or cancer. The consensual definition of cancer or neoplasia is a class of diseases that results from abnormal, uncontrolled proliferation of genetically altered cells that invade and destroy adjacent tissues. The genetic abnormalities (e.g., mutation, translocation, etc) of transformed or tumor cells can be caused by physical (e.g., radiation), chemical (e.g., carcinogens) or infectious (e.g., oncoviral) agents. Neoplasia can be benign, which means that cells abnormally proliferating, or tumors remain localized and do not invade other tissues. For the purpose of this review, I will call neoplasia any abnormal growth or tumors for which there is no evidence of metastasis. In contrast, neoplasia can spread or metastasize to other parts of the body of an organism; in this review, this will be designated as malignancy. In mammals, it is now well accepted that malignancy is a multistep process that requires six essential alterations in cell physiology: (i) self-sufficiency in growth signals; (ii) insensitivity to antigrowth signals; (iii) evasion of apoptosis; (iv) unlimited replicative potential; (v) sustained angiogenesis; and (vi) tissue invasion and metastasis. Many genes and pathways critically involved in neoplastic transformation and metastasis are evolutionarily conserved. Indeed, some forms of cancers are relatively widespread in all multicellular animals or metazoan, whose evolutionary origin is estimated to be more than 900 million years ago (MYA). In fact, non-mammalian model organisms such as Xenopus, Drosophila and Caenorhabditis elegans are contributing significantly to understanding tumorigenesis. In the early 20th century, Ehrlich first proposed the existence of immune surveillance for eradicating nascent transformed cells before they are clinically detected. Fifty years later, Burnet and Thomas postulated that the control of nascent transformed cells may represent an ancient immune system that played a critical role in surveillance against malignant transformation. Besides earlier experiments using drugs or irradiation to impair the immune system, more direct evidence of the crucial role of the immune system in preventing malignancies has been obtained using mouse strains deficient for specific genes controlling adaptive immunity such as Rag 2, Interferon $\gamma$ or Baft. Studies in mice also suggest a role for elements of the innate immune system such as type I interferon and NK cells in immune surveillance. In human, analyses of clinical data show strong correlations between immunosupression and cancer incidence. the type, density, and location of immune cells recorded in an extensive study of human colorectal tumors were found predictive of clinical outcome. While, evidence consistent with the concept of cancer immune surveillance is relatively well documented in mammals, especially in the mouse model, the importance of this role of the immune system in survival and natural selection is usually overlooked. In contrast to information concerning tumor occurrence and tumorigenesis, little is known about the role of the immune system in detecting and controlling neoplastic diseases in animals other than selected mammalian and avian species. This is of relevance since recent studies in mice and humans have stressed the multiple and complex interactions that occur between malignant cancers and their hosts. While the immune system maintains a surveillance that is crucial for detecting and preventing tumors, it may also establish selective pressures that shape and even may generate new variants that escape the immune system and display increased tumorigenicity and metastatic potential (immunoediting). So far, evidence in support of the process of immunoediting comes mainly from mouse studies comparing the properties of tumors induced by chemical carcinogen in animals that are either immunocompetent or immunodeficient (e.g., Rag2or perforin-deficient). Tumors obtained from immunocompetent mice are more tumorigenic and, more modified than those that have developed under low or absent immune pressure. As in immunesurveillance, the innate immune system appears also involved in shaping tumor. For example, NKp46, a specific NK killer receptor recognizing unidentified tumor ligands, can mediate mechanisms of tumor editing.

Although evidence in mouse and human collectively support immune surveillance and immunoediting concepts, there are still many unanswered questions. For example the respective contributions of the adaptive (e.g., T cell responses) and innate (e.g., pro-inflammatory responses) immune systems is unclear. How early during tumorigenesis the immune system can detect transformed cells and how efficient it is at these early stages to eliminate tumor cells is still an active area of research. In addition, the generally accepted assumption that malignancies with metastasis or true cancers are tightly linked to the adaptive immune system of jawed vertebrates, and the corollary that associates the lack of adaptive immunity in invertebrates with the rarity and benignity of neoplasia are perhaps a bit too premature and simplistic.

it could be useful to reevaluate the available evidence of tumor occurrence and severity in invertebrates and jawless vertebrates whose immune systems are fundamentally different from those of mammals. In fact, even ectothermic vertebrates that share a similar adaptive immune system with mammals may provide 
important clues on the importance of cancer immune surveillance. The objectives of this review are first to critically evaluate the evidence for neoplasia and malignancy in the animal kingdom from invertebrates to vertebrates, then to examine the information available about anti-tumor immunity in the different classes of animals, with a special focus on the frog Xenopus, and finally, to try to draw a synthesis based on comparative and evolutionary views of the intricate interactions between immune systems and cancer.

\section{Evidence of neoplasia in metazoan}

Invertebrates: The metazoan or multicellular animal kingdom is estimated to have emerged on earth more than 1,300 million years ago (MYA), and the separation of organisms without symmetry from those with radial symmetry, or with bilateral symmetry (bilaterian) occurred about 900 MYA. Remarkably, and despite some confusion in the literature about the diagnostic distinction between pseudoneoplasia and real neoplasia and other types of deregulated proliferation resulting from wound repair or infection (e.g., granuloma caused by mycobacterial or mucosal infection), diseases with the characteristics of neoplasia have been reported in several invertebrate species including species evolutionary very distant from vertebrates. Furthermore, increasing evidence suggests that genes and pathways critically involved in tumorigenesis are all conserved in the entire metazoan kingdom. We succinctly review here some salient examples. Freshwater and marine sponges (phylum: Porifera) are among the most distant relatives of vertebrates (chordata). These are diploblastic invertebrate organisms (e.g., formed by only 2 primary germ layers: the ectoderm and endoderm) without clear body symmetry (asymmetric). Although, to date, no well defined neoplastic disease has been reported in this phylum, the response of their cells to mitogens such as phorbol miristate acetate, and the presence of pro-oncogenes including ras, src and ets, suggest that oncogenic transformation is possible.

Corals are part of another major phylum distant from chordata, the cnidarians. These diploblastic organisms have a radial symmetry and are also mostly marine. A relatively well described disease known as calicoblastic neoplasm affects several species of corals of the Acroporidae family. These apparent tumors form slightly hemispherical protuberances that are characterized by fewer numbers of polyps per surface area, fewer zooxanthellae per polyp, and finer skeletal structures than normal. Tumorlike formation appears localized, stable and non-epidemic, but tumorous tissue appears to have suffered a higher mortality than normal tissue. The mechanism triggering tumor formation remains unknown, and some have interpreted the lack of evidence of anaplasia or mitotic figures (common but not necessarily required morphologic indicators of neoplasia) as growth anomalies than neoplasia. Like the Porifera, several genes and pathways involved in malignancy have been characterized in cnidarians. This includes the identification and molecular function of the p53 tumor suppressorlike protein nvp63 in a non-bilaterian animal, the starlet sea anemone Nematostella vectensis.
Nematodes or roundworms are a diverse phylum of triploblastic pseudoceolomate animals with more than 80,000 different species. The nematode C. elegans is a well-known model organism for which a tumor from the germinal layer induced by a member of the Notch family has been described. More recently, a homologue of the retinoblastoma susceptibility gene ( $\mathrm{Rb}$; also known as $\mathrm{Rb} 1$ ) that is functionally inactivated in most human solid tumors, has been characterized in C. elegans and shown to play a role in cell proliferation and differentiation. It is noteworthy that C. elegans has become a useful model organism to dissect critical genes and pathways affected during tumorigenesis such as apoptosis and Ras signaling.

Mollusca (e.g., mollusks) is one of the 2major phyla of triploblastic protostome animals. Many different tumors have been described in mollusks with either single or double shells. A number of examples of tumors with some malignant features exist, for example, oyster epithelioma, neurofibromas, sarcomas, and leukocytic neoplasms (leukemia-like disorders). A type of cancer similar to leukemia is well described in the mollusk Mya arenaria, a bivalve. These putative malignant leukemia cells are polyploid, transplantable, and can be grown in vitro. Although it is a matter of debate whether this type of tumor can be considered metastatic it is clearly invasive. Hemic neoplasia characterized by the presence of single anaplastic cells with enlarged nuclei and frequent mitosis, in hemolymph vessels and sinuses in the connective tissue are also found with increasing prevalence in the blue mussel Mytilus trossulus from polluted areas of Nakhodka Bay in the Sea of Japan and the Black sea. Besides the role of environmental "stressor", evidence suggests the involvement of mutations of homologs of the ras oncogene and deregulated expression of p53 tumor suppressor. Arthropoda (insects, crustaceans) is the second major triploblastic protostome phyla of which insects represent by far the largest number of animal species (more than 500,000). In insects, several types of tumors have been reported, especially in the fly Drosophila melanogaster, including neuroblastoma, ovarian, and imaginal disk tumors. the incidence of tumors of the testis and gut in D. melanogaster increases with age. Mutations affecting asymmetric division and the centrosome function of stem cells promote tumorigenesis. larval brain cells from transgenic lines of D. melanogaster with extra centrosomes can generate metastatic tumors when they are transplanted into the abdomen of wild-type hosts. The metastatic potential of tumors in D. melanogaster is also supported by the conservation of molecular control of cell migration (e.g., SAP97, TGF pathway) involved in mammalian metastasis. a large number of mammalian homologs of tumor suppressor genes and other genes controlling proliferation and migration (e.g., Serine/ threonine-protein kinase LATS1, Ras signaling, Raf/MAPK pathway) have been identified and characterized in D. melanogaster ; this further supports that the tumori-genesis process is conserved in this species, and that spontaneous neoplasia and even malignancies are at least possible.

Neoplasia in other arthropods such as crustacean are less well documented and are claimed to occur less frequently than in 
insects. The best described cases are a lymphoma-like neoplasm in the white shrimp, a decapod that may result from viral infection; and a putative carcinoma-like neoplasm in the hindgut of the red king crab, Paralithodes camtschatica. It is noteworthy that decapod crustaceans living in polluted areas postulated to induce neoplasia in mollusks, don't show similar diseases, and that experimental exposure to water-born carcinogens at doses known to induce tumors in fish and mollusks are ineffective in decapods such as the white shrimp.

In the group of deuterostomes, evidence of neoplasia in echinoderms is weak. review of the early literature by Wellings does not reveal any definite case of neoplasia among echinoderms. According to Sparks the only possible neoplasms recorded in echinoderms are the tumor-like epidermal lesions consisting of densely packed cells (mainly melanocytes and spherulocytes) that were originally reported by Fontaine in the ophiuroid Ophiocoma nigra., similar tumor-like mass of cells with brownish pigmented granules frequently occur within echinoderm tissues and can also correspond to unwanted material, mostly degenerating ceolomocytes, in the process of being eliminated. Another putative tumor observed in Holothuria leucopilota is more likely to be an unusual outgrowth of the ventral hemal vessel of the holothuroid gut multiple examples of neoplastic diseases in diverse invertebrates exist, but definitive evidence in this group of spontaneous metastasis such as a clear invasion of multiple tissues by tumor cells is rare. Possible exceptions may be found in mollusks and drosophila where the molecular control of cell migration present in mammals is conserved, and experimental metastasis have been obtained by transfer of mutated cells with asymmetric division. Another peculiarity of neoplastic diseases reported in aquatic invertebrates is that they are often associated with environmental stressors and pollutants (viral etiology is rarely found). Finally, the incidence of neoplasia varies greatly among different taxa (from relatively frequent in insects and mollusks to rare or very rare in crustaceans and echinoderms). This should be interpreted as a warning for making generalizations too hastily.

1.1.1.Vertebrates: In Agnathans or jawless vertebrates, an extensive study has revealed a high incidence of hepatomas and other tumors in the gut, pancreas and kidneys of the Atlantic hagfish collected from the Gullmar Fjord (Myxine glutinosa); these are possibly related to the high content of the pollutant DDT in this location. Cysts and tumor-like lesions in the endocrine pancreas have also been observed in the river lamprey (L. fluviatilis). In jawed vertebrates, elasmobranchs (rays and sharks) have been mistakenly claimed by some to be tumor-free. In fact, they appear to be as susceptible as any other vertebrates to tumors, and a large variety of neoplasms and malignancies have been reported, including neuroblastomas, osteomas, ranal and heptic carcinomas, cutaneous fibrosarcomas and fiborma, metastatic, melanomas, adenocarcinomas, and lymphomas. In bony fish, presumably as the result of a greater scrutiny owing to their economical importance, there is a large body of information concerning neoplastic diseases Indeed, a wide diversity of neoplasia and malignancies matching those found in humans has been described in more than 200 different species. The causes for many of these tumors are often unclear. Environmental factors, especially in aquaculture may play a role in the genesis of some of them, whereas oncoviruses appear to be involved in several cases. Only a few extensively studied examples are considered here. Skin neoplasms in bony fish include tumors in pigmented skin cells such as melanoma but also erythrophoromas and iridophoromas that are not found in mammals [. Interestingly, several retroviruses have been found associated with neoplasia in bony fish. The best known is the Walleye dermal sarcoma virus (WDSV) that has been etiologically linked to the development of Walleye dermal sarcoma, a common skin neoplastic disease of walleye fish (Sander vitreus) in the United States and Canada. Another retrovirus has also been identified in association with an outbreak of leiomyosarcoma in the swim bladder of the Atlantic salmon. Additionally, a less well characterized virus-like agent is the possible cause of transmissible neurofibromatosis and neurofibrosarcomas (tumors of neural tissues) in the bicolor damselfish Stegastes partitus. A platyfish-swordtail (Xiphophorus) tumor system has even been used to study the etiology of cancer and reports have demonstrated that neoplasia in these fishes is due to aberrant regulation of certain genes during the multistep process of cancer formation. In addition, model animals such as zebrafish display conserved genes and pathways involved in tumorigenesis and metastasis.

As is the case for most animals other than mammals, literature pertaining to amphibian neoplasia is scattered, often incomplete, and difficult to interpret owing to inconsistencies in diagnosis, nomenclature and misdiagnosis of infectious and inflammatory conditions. Among interesting cases to report is a spontaneous epithelioma of the Japanese newt, Cynops pyrrhogaster, that is of possible viral origin. Other neoplasia in different Urodelean (salamanders) and Anuran (frogs and toads) species have been reported These include pancreatic and renal carcinoma. From a large study of more than 1,200 animals, various spontaneous skin tumors have been described in Rana temporiaria and R. ridibunda. Mast cell tumors (mastocytomas) have also been found in a number of A. mexicanum and A. trigrinum (tiger salamander; [66]). These leukocytic tumors were located in the skin and invaded underneath tissues such as muscles of relatively aged inbred animals. Melanomas have been described in axolotls, and interestingly, can be induced by regeneration. One notable type of adenocarcinoma, the Lucké tumor, found in Rana pipiens is among the first documented case of a virally-induced tumor in vertebrates.

Despite the wide use of the South African clawed frog Xenopus laevis as an experimental animal model, there are only a few reports of spontaneous tumors in this species. In addition, the prevalence, etiology and pathogenesis of these diseases are not well established. To highlight some of the confusion in the literature pertaining to the true diagnosis of neoplasia in amphibians, it's worth mentioning a pseudotumor, namely an infectious granuloma that was originally reported as a spontaneous malignant lymphosarcoma of viral origin. Subsequent to that report, no virus 
particles could be detected by electron microscopy, and thanks to a series of transplantation studies, the X. laevis tumor-like growth was found to be a transmissible, but not transplantable, bacteriainduced infectious granuloma. This case notwithstanding, there is no doubt that like other vertebrates Xenopus is affected by a whole variety of true neoplastic diseases including tumors that metastasize. Adenoma of the liver appears relatively frequently. It is usually detected during dissection as a markedly enlarged liver lobe that can reach twice its normal size. In most cases, further diagnostics are missing. In our large X. laevis colony, we estimate that such hepatomas affect about $5 \%$ of our animals per year. Notably, spontaneous teratomas containing significant thyroid carcinoma components have been described in X. laevis. These teratomas were associated with developmental defects in multiple sibling frogs suggesting a genetic basis for this disease. Other spontaneous neoplasias in X. laevis include tumors of the digestive track, melanophoromas, and more rarely, renal carcinoma and nephroblastoma. We have recently described a type of ovarian tumor in X. laevis [2]; a similar tumor was also described in another anuran species (Leptodactylus fallax;).

A particularly interesting type of malignancy studied in X. laevis is a lymphoid tumor. Over the course of about a year, a series of spontaneous thymic lymphoid tumors occurred in the Xenopus colony of the Basel Institute of Immunology. Several stable tumor lymphoid cell lines (named B3B7, 15/0, 15/40 and ff-2) were derived from these frogs. The availability of these tumor lines as well as genetically defined MHC compatible inbred strains and clones of X. laevis have provided the unique opportunity to study both tumorigenesis and anti-tumor immunity in an amphibian. Metastasis in the original animals as well as in some compatible recipients in which tumor cells were transplanted was unequivocally observed. A similar type of thymic tumor was described in another laboratory, and another type of spontaneous leukocytic, possibly monocytic, tumor very different from the thymic tumors mentioned, was recently reported by Du Pasquier et al.. Finally, although virusinduced neoplasia has not been documented so far in X. laevis, an endogenous retrovirus has been found that is closely related to the epsilon-retroviruses WDSV and WEHV types 1 and 2, that are associated with neoplasia in walleye fish.

In reptiles, among the large variety of neoplasias reported, one can mention a metastatic oviduct adenocarcinoma in snakes (e.g., Boa), and a fibropapillomatosis likely caused by a herpes virus in sea turtles. Herpesvirus-associated papillomatosis has just recently been described in the green lizard, Lacerta viridis,. Lymphosarcoma and renal neoplasia have also been observed. Interestingly, evidence of tumors has been found in dinosaur fossil records from the Cretaceous. These neoplasia included hemangiomas and metastatic cancer, desmoplastic fibroma, and osteoblastoma. The literature on malignancies in endothermic (warm blooded) vertebrates (e.g., birds and mammals) is obviously far more abundant and these diseases are better characterized than in invertebrate and ectothermic (cold blooded) vertebrate species. Almost 100 years have passed since Peyton Rous found that a sarcoma in chickens could be transmitted by cell-free extracts of tumor tissue. The identification of the Rous sarcoma virus (RSV) that induces tumors of the connective tissues (sarcomas) initiated a new field of research leading to the discovery of oncogenes. In birds, various other oncogenic retroviruses cause neoplasia and leukemia. For example, Marek's disease (MD) is caused by a ubiquitous lymphotropic alphaherpesvirus, MD virus (MDV). This disease is characterized by a variety of clinical signs among which are neurological symptoms, chronic wasting and, most notably, the development of multiple lymphomas that manifest as solid tumors in the viscera and musculature. The first successful anti-tumor vaccine has been developed against MD, although vaccine efficacy has decreased concomitantly with the increase in virulence of the virus. Other retrovirus-caused neoplasias include avian leukosis virus (ALV), reticuloendotheliosis virus (REV) and lymphoproliferative disease virus (LPDV).

Given the high incidence of tumors and the volumes of research reported in mammals, only 2 interesting types of neoplasia will be mentioned here, because of their mode of transmission and apparent lack of immunogenicity. The Tasmanian devil facial tumor disease (DFTD) and the canine transmissible venereal tumor (CTVT) are the only known naturally occurring clonally transmissible cancers. In dogs, DFTV is a fatal monophyletic clonally transmissible tumor that is basically an allograft transmitted between devils by biting. Phylogenetic analyses indicate that CTVT most likely originated from a wolf or an East Asian breed of dog between 200 and 2500 years ago. An interesting common feature of these 2 types of tumors is the apparent lack or poor immune response of the mammalian host. In summary, in the entire vertebrate subphylum there is abundant evidence of a large variety of malignancies associated with metastasis. In addition, many of these malignancies are virallyinduced 2.

Tumor immunity: The characterization of immunity in the whole animal kingdom has recently made marked progress in parallel with the progress in expressed sequence tag (ESTs) data banks, and whole genome sequencing and analysis for an increasing number of organisms (http://www.ncbi.nlm.nih.gov/ genomes/leuks.cgi). In particular, the use of short-range synteny relationships, or gene neighboring, has provided a powerful way to identify many immune genes that were hard to find by molecular techniques because of their sequence divergence. This information has led to new insights about the diversity and complexity of immune systems in various animals. This is particularly true for invertebrate immunity, usually referred to as innate immunity, which involves a wide variety of cells and pathways that globally target pathogens. In contrast to molecular, phylogenetic and to some extent expression patterns, comparative functional analysis of immune genes is still mainly under development. As such, investigation of the role of the immune system in detecting and controlling neoplasia is still poorly investigated in species other than X. laevis, chickens and mammals. A general presentation of the immune systems of invertebrates and vertebrates is beyond the scope of this review and can be found elsewhere. This discussion will focus on aspects related to 
tumor immunity, and only in invertebrates and nonmammalian vertebrates.

Invertebrates: A large body of data indicates that invertebrate marine organisms produce a multitude of active substances that, in humans, exhibit potent anti-tumor activity; these include alkaloids and terpenoids. Peptides named alloferons isolated from diptera (insects) also have anti-tumor capacities when tested on mammalian cells., it is not clear whether these different substances are produced in response to neoplastic transformation and if they can effectively control self-tumor development in the organisms that produce them. In drosophila, hemocytes have been shown to adhere to tumors upon detection of basement membrane disruption and subsequently counter their growth. Furthermore, these anti-tumor responses involve JAK/STAT-activating cytokines.

Concerning potential cell-mediated anti-tumor immune responses in invertebrates, NK-like cell mediated cytotoxicity has been described for some invertebrate species including sponges, annelids mollusks, insects and sea urchins. Although immune recognition in invertebrates is not based on MHC molecules, C-type lectin-binding molecules closely similar to vertebrate CD94 and NKR-P1 (typical NK cell markers in mammals) have been identified in urochordates. A CD94 homolog was also identified in hemocytes of the tunicate Ciona intestinalis. In fact, in addition to receptors specific for MHC class I molecules, mice and human NK cells also possess inhibitory receptors specific for non-MHC ligands such as E-Cadherins, lectins or collagen. Since these families of molecules are present in all metazoans, their interaction with NK cells and their involvement in anti-tumor NK cell responses in invertebrates is possible. It is important to note, that the cytotoxic activity in all the studies reported so far in invertebrates has been characterized in the context of either alloreaction or using as cell targets mammalian (mouse, human) tumor cell lines that are especially sensitive to NK killing in vitro. the ability of invertebrate NK-like cells to kill invertebrate cognate tumor cells, as well as the active involvement of these effector cells in in vivo host responses against tumor still remain to be demonstrated. Other immune defenses in invertebrates include phagocytosis, encapsulation, and in arthropods, melanization. Although these effector functions can play a critical role in anti-tumor responses, their involvement has not been formally studied.

A last point to consider is that although the immune systems of invertebrates are usually considered to consist of immune receptors and effector molecules that are of limited diversity and germ-line encoded specificities, "adaptive somatic diversification" of molecules involved in immune defense has arisen as well, apparently by convergence, in drosophila, snails and sea urchins. Increasing evidence suggests that these surprisingly diversified repertoires of immune molecules are involved in non-self recognition. Whether these immune elements play a role in immune responses to neoplasia remains to be determined.

Nonmammalian vertebrates: A review of mammalian tumor immunity, a very active area of research especially in mice and humans, is beyond the scope of this paper; many reviews are already available. this review will focus on evidence of tumor immunity in ectothermic vertebrates and birds. Unfortunately, in jawless fish (Agnatha), virtually nothing is known about tumor immunity despite the substantial record of malignancy occurring in lamprey and hagfish. NK or other types of cell-mediated cytotoxicity has not been reported so far in this taxon. this may soon change. The capacity to generate a clonally diverse anticipatory repertoire of lymphocyte receptors was, for a long time, thought to be the prerogative of jawed vertebrates. Recently,, a remarkable convergent, adaptivelike immune system that is based on somatic recombinatorial assembly of leucine-rich-repeat genetic modules encoding variable lymphocyte receptors has been discovered both in lamprey and hagfish. the cells producing these immune molecules have the morphology of lymphocytes with characteristics analogous to the $\mathrm{B}$ and $\mathrm{T}$ cells of jawed vertebrates. Given the postulated complex interactions between tumors and the adaptive immune system of jawed vertebrates, it would be highly relevant to explore the immune response to tumors in these agnathan species.

In contrast to jawless vertebrates, the innate and adaptive immune systems of jawed vertebrates are fundamentally conserved from elasmobranchs to mammals. In particular, genes involved in antigen processing and presentation (e.g., MHC class I, class II, immunoproteasome) and generation of somatic repertoires of lymphocyte receptors (e.g., Rag, Ig, TCR $\alpha, \beta, \gamma, \delta$ ) are all present. other than Xenopus that will be examined in the next section, investigation of anti-tumor immune responses has not yet been formally investigated in any ectothermic vertebrate.

In elasmobranchs, both cell-mediated spontaneous and antibody-dependent killing has been characterized in the nurse shark. the anti-tumor potential of these types of killing, even against mammalian targets, has not been evaluated. More generally, evidence for the role of the adaptive immune system either direct (e.g., characterization of anti-tumor $\mathrm{T}$ or $\mathrm{B}$ cell responses) or indirect (increased prevalence of tumors with age) is completely lacking in this taxon.

In bony fish (teleosts), NK-like and other less well defined types of cell-mediated cytotoxicity has been reported in several species including carp and zebrafish. One of the more promising models for functional studies of cell-mediated cytotoxicity is the catfish where a unique in vitro culture system has been designed to generate stable leukocyte cell lines, and in which both NK and CD8 T cells have been characterized molecularly and functionally albeit not in anti-tumor responses. Investigations of anti-tumor immunity in this animal model would likely provide important information. Although genes encoding CD8 and CD4 as well as many relevant cytokines have been characterized in several fish species, nothing is known about anti-tumor T cells or immune responses to virallyinduced neoplasia in bony fish. Similarly, type I interferon and other related pro-inflammatory responses against pathogens is an active area of study in several model and economically important fish species. the possible involvement of these systems in immune surveillance and anti-tumor responses awaits investigation. 
To date, the amphibian Xenopus is the only ectothermic vertebrate species where the study of anti-tumor responses has been formally investigated in vitro and in vivo thanks to the unique collection of thymic lymphoid tumors. Earlier studies with the ff-2 lymphoid tumor that is transplantable and tumorigenic in tadpoles and young post-metamorphic froglets with a relatively poor $\mathrm{T}$ cell effector system, but not transplantable in fully mature adults unless they are $\mathrm{T}$ cell deficient (thymectomized at early developmental stage, or sublethaly $\gamma$-irradiated) provided the first clear evidence of the conserved critical involvement of T cells in tumor immunity. Further studies, using the $15 / 0$ tumor that is transplantable and very tumorigenic both in larvae and adults that share the same MHC alleles $(\mathrm{a} / \mathrm{c})$ with the tumor, have demonstrated that NK cells, CD8 $\mathrm{T}$ cells, and other less well-characterized cell types are responsible for generating anti-tumor immune responses in Xenopus.

The active role of NK cells in vivo against transplanted tumors was demonstrated by pre-treatment, one day prior to tumor challenge, with the Xenopus-specific anti-NK cell monoclonal antibody $(\mathrm{mAb})$ 1F8. This resulted in accelerated appearance of tumors as well as an increase in the rate of tumor growth. In addition, potent NK cytotoxic activity has also been demonstrated in vitro against both 15/0 and B3B7 thymic lymphoid tumor cells that do not express classical MHC class Ia proteins. The phenotypic and functional characterization of these anti-tumor NK cells in spleens reveals that they are large granulated cells that express the NK surface marker 1F8, but not surface Ig and CD8 molecules, and that they also differentiate in frogs that had been thymectomized at an early developmental stage and rendered T-cell deficient. Their cytotoxic activity requires stimulation by T-cell derived growth factors thereby highlighting the importance of $\mathrm{T}$ cells and their secretory products for the development of a potent anti-tumor immune response. Mammalian NK cells are activated through the cumulative signals integrated by their cell surface activating and inhibitory receptors. Ligands for NK cell receptors include MHC class I molecules, and either their absence or their deregulated expression on the surface of cells, can lead to the activation of NK cells. As in mammals, Xenopus NK cells do not kill cognate cell targets expressing MHC class I molecules (they kill class Ia negative 15/0 tumor cells derived from the LG-15 Xenopus clone but not class I+ splenic lymphoblasts). 15/0 tumors express several nonclassical MHC class Ib (class Ib) gene products that require association with $\beta 2$-microglobulin $(\beta 2-\mathrm{m})$ for efficient surface expression. Notably, 15/0 tumor cells whose class Ib surface expression is impaired by RNA interference targeting $\beta 2-\mathrm{m}$ or class Ib, are more susceptible to NK cell killing in vitro. These data suggest that Xenopus NK cells express inhibitory receptors that can interact with class Ib molecules. Although Xenopus has only a few genes encoding killer inhibitory (KIR) molecules, there is a large family of Fc-like receptors most of which are of the inhibitory type with an immunoreceptor tyrosine-based inhibitory motifs (ITIM) in their cytoplasmic tail.

Similar to NK cells, the role of T cells in anti-tumor responses was first evidenced by immunization with the heat shock proteins (hsps) gp96 or hsp70 purified from 15/0 tumor. As in mammals, Xenopus hsps can chaperone antigens from 15/0 tumors and elicit an anti-tumor cellular response with potency, specificity and kinetics typical of a T cell response. Particular involvement of CD8 T cells in tumor immunity was revealed in vivo by antibody depletion that accelerated the development of transplanted 15/0 tumors. Further evidence was obtained by adoptive transfer of CFSEstained splenocytes from hsp-immunized Xenopus clones into naïve recipients of the same clone that were subsequently challenged with $15 / 0$ tumors. Significant proliferation of adoptively transferred primed CD8 T cells, but not naïve control ones, was detected by the dilution of the CFSE dye using 2-color flow cytometry.

Although, in Xenopus hsps are able to elicit conventional class Ia-restricted CTL responses against minor histocompatibility $(\mathrm{H})$ antigens, hsp-mediated CD8 $\mathrm{T}$ cell responses against the 15/0 tumor cannot be class Ia-restricted since this tumor does not express class Ia molecules. We have further characterized these anti-tumor class Ia-unrestricted CD8 T cells (CCU-CTLs) and shown that they interact with class Ib molecules. This was achieved by RNAi silencing of either $\beta 2$-m or directly class Ib expression, which results in a knockdown of class Ib surface protein on the 15/0 tumor. This treatment resulted both in an impairment of the CD8 T cell's ability to recognize and kill this tumor in vitro and in the increased tumorigenicity of the class Ib-silenced tumor clones in vivo. These experiments point to the critical role of CD8 T cells as anti-tumor effector cells in Xenopus and also demonstrate that at least a subset of these effector cells can interact with class Ib molecules to mediate anti-tumor immune responses.

Last but not least, some indirect evidence suggests that in Xenopus, NKT-like cells may also be involved in anti-tumor immune responses. In mouse and human, despite their small number relative to T and NK cells, NKT and so called CTL-NK cells appears to contribute significantly in anti-tumor responses either directly or through regulation of other anti-tumor effectors. In Xenopus as stated earlier, in vivo treatment with the anti-NK cell mAb 1F8 impairs rejection of transplanted tumor cells. Importantly, this NK cell-associated molecule is also expressed by a minor population of CD8 T cells in which fully rearranged TCR $\beta$ mRNA can be identified. Approximately $4 \%$ of adult splenocytes co-express CD8 and the NK cell-associated molecule 1F8. in Xenopus, a population of cells exists that expresses both NK- and T cell-associated markers. In addition to inhibiting NK cells, in vivo treatment with the 1F8 mAb likely also impairs NKT-like cells in Xenopus. As in other ectothermic vertebrates antibody responses against tumors are sketchy. The only piece of data concerns the detection of antibody following immunization of frogs from the MHC homozygous partially inbred F line with the thymic lymphoid tumor ff- 2 against a large surface molecule (180-200 kDa) that is also recognized by anti-F alloserum.

Endothermic vertebrates: A review of mammalian tumor immunity, a very active area of research especially in mice and humans, is beyond the scope of this paper; many reviews are already available. this review will focus on evidence of tumor immunity in 
chicken. The chicken is perhaps the best non mammalian vertebrate model that has been characterized in terms of the immune response, the genetics of disease resistance and vaccine response, and the genomic structure and function of the MHC. With respect to antitumor immunity, studies with chickens have concentrated mainly on immune responses against onco viruses; this focus has resulted in the development of successful vaccines against tumors.

Particularly is the association of certain MHC haplotypes with resistance to virus-induced malignancies. The first of such association to be described, and still among the strongest known, is the association of the MHC haplotype B with resistance to Marek's disease. particular haplotypes are also associated with resistance of other oncogenic viruses including ALV. The strong association of MHC haplotypes and resistance to virus-induced malignancies is presumably related to the peculiarity of chicken MHC, which compared to the MHC of typical mammals, is much smaller and simpler, with a different genomic organization. Recombination is rare within the chicken $\mathrm{MHC}$, and this property has been proposed to allow coevolution between interacting genes, such as the class I, TAP1, TAP2 and tapasin genes. A consequence of this coevolution is the expression of a single dominantly expressed MHC class I molecule due to peptide-binding specificity and cell-surface expression level, which determines the immune response to certain infectious pathogens and explain the striking associations with resistance to these pathogens. recent genetic studies have revealed that allelic difference of BG1 an IgSF receptor-like protein located in the MHC locus that contains an immunoreceptor tyrosine-based inhibition motif (ITIM) plays a major role in Marek disease and other oncogene-induced lymphomas. the association of MHC haplotypes with resistance to oncoviruses is not only due to antiviral responses but also to a better ability to control tumors growth and metastasis. vaccination with low oncogenic RSV strains induces not only protective immunity but also results in regression of visceral tumors and erythroblastosis induced by RSV. Finally, contribution of non-MHC genes in resistance to viral replication and tumor growth is also documented.

Given both case reports and experimental data, there is little doubt that neoplasia is widespread in the whole animal kingdom from sponges to man. The risk of uncontrolled proliferation appears to be a condition of multicellularity and differentiation. The evolutionary emergence of genes controlling differentiation programs and cell proliferation has provided the way for their disruption and deregulation through mutations and escape by cell transformation. The increase in complexity of animals should have offered more possibility for neoplastic transformation. Considering the potential danger of neoplastic transformation for survival, it is reasonable to postulate that any ability to detect and control such tumor cells would provide a selective advantage, and that neoplasia may have played an important role in the evolution of the immune system as much as pathogens. like host-pathogen interactions, the intricacy of interactions between tumors and host immune systems can be viewed as the result of their "arms race" during evolution that increased in complexity and degree of specialization in parallel with the diversification of organisms.
It is generally considered that neoplasia is less frequent in invertebrates than vertebrates. Certainly, the information in the literature about neoplasia in invertebrates is scant. reports are more than 20 years old, and recent surveys of neoplasia are limited to organisms of economical importance. one has to remain cautious about any conclusion that can be drawn from such a small sample of data. The frequency of tumors in any invertebrate species may depend on the extent to which monitoring is performed. it is difficult to estimate the possible fraction of animals in the wild that die from a neoplastic disease and disappear from the environment. These caveats notwithstanding, it still appears that both the frequency and the diversity of types of neoplasia in invertebrates are less than in vertebrates, and in some invertebrate taxa such as echinoderms tumor are virtually absent.

when we look over the data available in marine and fresh water invertebrates, the major factors for neoplastic disease appear to be environmental stressors (e.g., pollutants), whereas there is little to no evidence of virally-induced tumors. It is possible that the aquatic environment is more favorable for long term exposure to chemicals acting as mutagens or directly affecting regulation of differentiation and proliferation. Owing to the identification of several oncoviruses in several fish species, it is more difficult to explain why similar viruses are not as frequent in invertebrates other than that they have not yet been identified. As already mentioned, neoplasia has been reported far more frequently in vertebrates than in invertebrates, and the diversity of tumor types is also greatly increased. a potentially more fundamentally important difference in neoplastic diseases between invertebrates and vertebrates is metastasis or real malignancy. With the possible exception of bivalves and drosophila, evidence of metastasis is absent in invertebrates, whereas malignancies have been frequently described in all vertebrates including elasmobranchs and agnathans. The particular interest in considering neoplasia in terms of differences in severity and its capacity to metastasize concerns the possible role of the immune system. It has been proposed that the adaptive immune system of jawed vertebrates is playing an active role in the malignancy process. According to this view, neoplasia in invertebrates, with a few exceptions being due to pollution, remain mostly benign and without differentiation of invasive tumor variants because the innate immune system is relatively inefficient in eradicating tumors and, does not establish a strong enough selective pressure. In contrast, the increased capacity of the vertebrate immune system to generate strong and specific anti-tumor responses ultimately results, over time, in the selection of tumor variants that escape immune surveillance and are more invasive.

A presumptive relationship between development of metastasis and pressure of adaptive immunity could also be drawn in jawless fish. Although the anti-tumor capacity of the agnathan's immune system is not known, it is noteworthy that its adaptive immune system is distinct and convergent to that of jawed vertebrates and that malignancies have been described. Lampreys and hagfish have an adaptive immune system that uses somatic diversification analogous to, but completely independent of, the adaptive immune 
system of jawed vertebrates. T-like cells express surface receptors generated by gene conversion. It is tempting to speculate that these T-like cell effectors can be involved in tumor immune surveillance, and as such, are involved in selection of tumor variants that ultimately escape the control of the immune system and invade other tissues, as is the case for jawed vertebrates.

Although spontaneous malignancies have not been reported in drosophila, the invasive nature of tumors resulting from the mutation of genes regulating asymmetric division constitutes clear evidence that metastasis could occur in this invertebrate. the immune system of drosophila and other dipterians, has been revealed to be more complex than previously thought. Not only do they have a conventional innate immune system that is armed by a large variety of receptors suggesting a refined capacity to detect antigens, but they also enjoy a huge potential for somatic diversification of DSCAM molecules generated by alternative splicing that show some features analogous to an adaptive immune system. not much is known about anti-tumor immunity in bivalves, rapid progress has been made in characterizing immune systems in mollusks including the large and diversified repertoire of molecules such as the family of fibrinogen-related proteins (FREPS) of the freshwater snail Biomphalaria glabrata. It is, not impossible that different groups of invertebrates are capable of more complex immune surveillance and responses than usually assumed. Rather than a simplistic dichotomy between invertebrates and vertebrates, one should perhaps consider in more detail for any given taxon or group of organisms, the potentiality of their immune systems in relation to the kind of neoplastic diseases that can occur. This consideration begs for obtaining more information about neoplasia in the animal kingdom. It will also be very relevant in the future to explore and investigate the possible involvement of viruses in tumorigenesis of ectothermic vertebrates (including the Agnathan) and invertebrates with a putative adaptive-like immunes system such as in the diptera [21].

Daniela Melillo et al: "The ability of developing immunological memory, a characteristic feature of adaptive immunity, is clearly present also in innate immune responses. In fact, it is well known that plants and invertebrate metazoans, which only have an innate immune system, can mount a faster and more effective response upon re-exposure to a stimulus. Evidence of immune memory in invertebrates comes from studies in infection immunity, natural transplantation immunity, individual, and transgenerational immune priming. These studies strongly suggest that environment and lifestyle take part in the development of immunological memory. in several instances the formal correlation between the phenomenon of immune memory and molecular and functional immune parameters is still missing. In this review, we have critically examined the cellular and humoral aspects of the invertebrate immune memory responses. In particular, we have focused our analysis on studies that have addressed immune memory in the most restrictive meaning of the term, the response to a challenge of a quiescent immune system that has been primed in the past. These studies highlight the central role of an increase in the number of immune cells and of their epigenetic re-programming in the establishment of sensu stricto immune memory in invertebrates[22]."

Aysu Uygur et al: "Adult humans fail to regenerate their hearts following injury, and this failure to regenerate myocardium is a leading cause of heart failure and death worldwide. all adult mammals appear to lack significant cardiac regeneration potential, some vertebrates can regenerate myocardium throughout life. new studies indicate that mammals have cardiac regeneration potential during development and very soon after birth. The mechanisms of heartregenerationamongmodel organisms, includingneonatal mice, appear remarkably similar. Orchestrated waves of inflammation, matrix deposition and remodeling, and cardiomyocyte proliferation are commonly seen in heart regeneration models. Understanding why adult mammals develop extensive scarring instead of regeneration is a crucial goal for regenerative biology. inducible Cre recombinase (CreER) expression was driven by the cardiac myosin light chain 2 ( $\mathrm{cmlc} 2$ ) promoter sequence, resulting in the expression of a loxP flanked EGFP reporter gene. Before cardiac injury, all cardiomyocytes expressing Cmlc2 were labeled by EGFP expression, and 30 days post ventricular resection, the majority of the regenerated tissue was labeled with EGFP expression, showing that cmlc2+ cardiomyocytes were the major source for regenerated myocardium.

Poss etal further dissected the contribution from subpopulations of cardiac muscle into the regenerate. They employed a multicolor clonal analysis sytem in order to identify 3 distinct muscle lineages in the zebrafish heart: primordial, trabecular, and cortical muscle, distinct in their order of development during cardiac morphogenesis. The multicolor clonal analysis technique employed in this study was adapted from the Brainbow technology that was initially developed in the mouse. Cre/lox recombination was used to create a stochastic gene expression pattern from 3 tandem fluorophores, allowing one to distinguish clones from a specific recombination event Clonal labeling in zebrafish cardiomyocytes revealed that during regeneration, proliferation of cortical muscle in the wound area is detectable at 14 days post amputation and constitutes the primary component of the regenerate wall, whereas the primordial layer of muscle is first detected at 30 days post amputation in a restricted lateral expansion, as observed during embryogenesis. the appearance of muscle layers during regeneration is in reverse order to the order in cardiac development. Comparable fate mapping analysis has also been performed in the neonatal mouse. Using genetic fate mapping and stable isotope imaging technology, Senyo et al showed during normal myocardial homeostasis in the adult mouse, new cardiomyocytes arise from pre-existing cardiomyocytes. after injury, modest new cardiomyocyte regeneration near the injury increases, and these myocytes appeared to arise from pre-existing cardiomyocytes. Kimura et al. reported fate mapping of cycling cardiomyocytes following injury to a subset of hypoxic cardiomyocytes that express HIF1- $\alpha$. The extent to which a progenitor cell pool contributes to cardiomyocyte renewal remains controversial: c-kit+ cells were 
previously reported to mark cardiomyocyte progenitors but a quantitatively rigorous genetic fate mapping study showed that this contribution is functionally negligible. Hatzistergos et al reported that c-kit+ cells are of cardiac neural crest origin in development and their limited contribution to cardiac progenitors is due to a nonpermissive environment in the developing heart. a new study of multiple lines of genetically engineered mice showed no significant cardiogenesis from c-kit+ cells, consistent with van Berlo et al study. The role of ckit+ cells in post-infarction myogenesis was explored in 2012 by Jesty et al, who reported that ckit+ cells partially support repopulation of the myocardium following injury in the neonatal heart, but that ckit+ cells do not adopt cardiomyocyte cell fate during myogenic repair in adult mice that harbor a transcription marker for ckit (ckitBAC-EGFP) A major difference between lower vertebrates such as the zebrafish and newt and mammals may be their ability to complete the cell cycle. In contrast to the mononucleated zebrafish cardiomyocytes that can reenter the cell cycle in adulthood, many of the cardio-myocytes in the mammalian heart become bi-nucleated either before or shortly after birth In rodents, up to $95 \%$ of cardio-myocytes are bi-nucleated while b-inucleation is much lower in human cardio-myocytes at 3040. A study by Bersell et al. showed that Neuregulin1 can induce proliferation of differentiated adult cardio-myocytes in cell culture and in vivo. Interestingly, Neuregulin1 (NRG1) appears to affect the mono-nucleated subpopulation of differentiated cardio-myocytes, supporting the premise that mono-nucleated cardiomyocytes may be more receptive to cell cycle reentry. D'Uva et al recently showed that cytokinesis in bi-nucleated cardio-myocytes are also possible with constitutive activation of the NRG1 co-receptor ERBB2. The curious role of nerves.

Over the last century, studies across multiple species has shown that nerves are indispensible for regeneration in many organs. experiments in newt limbs show that de-nervated limbs cannot regenerate. Newt and salamander lens, retina and tail are other systems where adequate number of nerve fibres are required to guide regeneration To date, several nerve-derived factors have been shown to function in regeneration, including fibroblast growth factors (FGFs) in vertebrate limb regeneration, glial growth factor (GGF) in zebrafish tail regeneration and vertebrate limb regeneration $\mathrm{nAG}$ in newt limb regeneration and glial-derived neuro-trophic factor (GDNF) in hematopoietic regeneration in mammals the role of nerves in heart regeneration has also been explored. Mahmoud and O'Meara et al. mechanically interrupted the left vagus nerve and found that this suppresses the heart regenerative response upon injury in neonatal mice. This suggests a role for parasympathetic nerve function in heart regeneration. In another recent study, White et al. explored the influence of sympathetic nerves in cardiac regeneration. Mice that undergo chemical sympathec-tomy have inhibited sympathetic regrowth and failed cardiac regeneration following apical resection surgery. These new findings suggest that nerves may function in the regenerative process, although this does not appear restricted to sympathetic vs. Parasympathetic nerves. It is possible that a critical density of nerve factors is necessary to support regeneration.
The concept of a critical nerve density rather than specific nerve synaptic activity in driving regeneration is consistent with findings in axolotl limb regeneration. Thus, regulation of the regenerative response in many tissues by nerves appears to be an evolutionarily conserved pathway among different species including lower vertebrates, and the study of nerves in cardiac regeneration could shed light into conserved regenerative molecular pathways.

Inflammatory and immune response in heart regeneration: Unlike embryonic development, tissue growth in regeneration is initiated by an injury, and the inflammatory response to that injury is a critical regulator of the regenerative process. Inflammation can drive regeneration but can also inhibit it under some circumstances. Godwin et al. reported that following macrophage depletion, newt limb regeneration fails and leads to extensive fibrosis. In contrast, evidence for the constraining effects of a developed immune system on regeneration come from studies of Xenopus limbs. As young Xenopus larvae transition into adulthood through metamorphosis, their immune system also passes through gradual maturation; concominantly, they lose their regenerative capacity Grow et al. performed gene expression analysis comparing earlier stage regeneration-competent xenopus limbs and later stage regeneration-incompetent xenopus limbs and reported a higher level of pro-inflammatory genes expression 1 day post amputation in the regeneration incompetent limbs Limb amputation experiments in the developing xenopus limb suggest that it is the local inflammatory response subsequent to the wound formation that exerts a constraining effect on the regenerative capacity. the studies in frogs and salamanders show that the early inflammatory response plays a crucial role in the intiation of regenerative processes.

In lower vertebrates and mammals, cardiac injury is also associated with an initial wave of inflammation. The consequences of the inflammatory/immune response is remodeling through scarring in non-regenerating animals, versus re-modeling through cellular repopulation in re-generating animals. Infiltration by inflammatory cells in injured heart peaks around 3 days postamputation in zebrafish hearts The inflammatory system responds immediately to cardiac injury in all vertebrate species studied In mice, monocyte and macrophages are required for cardiac regeneration, and injury-induced cardiac proliferation is inhibited by immunosuppression Acute inflammation is required for neonatal heart regeneration, and in the absence of interleukin 6 (IL-6), cardio-myocytes fail to proliferate upon injury.

The relationship observed in anurans between maturation of the immune system and the decrease in regenerative capacity has been explored in mammals in the context of postnatal changes in the immune system and the loss of capacity for cardiac regeneration Aurora et al. identified differences in the cellular immune response to MI in 1 day old and 14 day old mice; they used a macrophage depletion model in neonatal mice to show that macrophages are required for regeneration and neo-angiogenesis in the injured heart. Interestingly, macrophage depletion did not influence cardiomyocyte proliferation following infarction. The molecular profiling 
of macrophages suggested that secretion of pro-angiogenic cytokines may be responsible for their important role in cardiac regeneration.

Angiogenesis and heart regeneration: Throughout phylogeny, formation of new vasculature following injury is vital for re-generation. As new tissue with complex architecture is restored, the Re-generating tissue needs a continuous supply of energy and substrates as well as routes for eliminating metabolic products. Therefore, a functioning dynamic vasculature is critical for a successful regenerative response. In the absence of neovascularization following injury, the zebrafish heart fails to regenerate and instead forms extensive fibrotic scarring. Gene expression and genetic analyses in zebrafish have established regulators of angiogenesis that are essential for the response to cardiac injury. FGF receptor expression in the epicardium and FGF ligand expression in the myocardium appear to be required for formation of new vasculature, and this process is thought to regulate epithelial to mesenchymal transition (EMT) of the epicardium in order to form coronary vasculature, Additionally, Pdgf signaling is required for epicardial proliferation and new blood vessel formation in cardiac regeneration, suggesting that new vascular formation during regeneration recapitulates molecular mechanisms that govern developmental processes of blood vessel formation. Interestingly, cxcr4a mutant zebrafish that fail to develop coronary vasculature in the myocardium can be viable as adults, but the mutants that survive into adulthood fail to regenerate their hearts, suggesting loss of this oxygen supply is especially critical for regeneration.

In neonatal mice, robust neovascularization is evident during the regenerative response to either apical resection or MI injuries. In adult mice where the regenerative response is not evident, the neo-vascular response is also not observed, suggesting that lack of blood vessel formation after injury could contribute to loss of cardiac regenerative capacity. Factors inducing vascular regeneration have been shown to improve tissue renewal and cardiac functional restoration in adult mice following MI. Zangi et al. have employed modified RNA (modRNA) technology to induce VEGF expression in a spatiotemporally controlled manner and thereby improve the regenerative response following injury, through neo-angiogenesis.

Extracellular matrix and heart regeneration: During normal cardiac development, signaling from extracellular matrix (ECM) provides structure and guidance for cellular migration, proliferation and differentiation. For example, fibronectin is a key cue for migration of cardio-myocytes towards the midline and is a regulator of cardio-myocyte proliferation. Similarly, changes in the tissue microenvironment comprise a crucial component of the regenerative response. In neonatal mice and lower vertebrates, the myocardium goes through an extensive remodeling process and scar formation is minimized compared with adult mammalian myocardium, but new extracellular matrix deposition is nevertheless considerable. Ablation of transient scar formation in zebrafish through pharmacological inhibition of TGF $\beta$ signaling abolishes cardiac regeneration Studies in the newt have revealed that an increase in ECM components precedes cardio-myocyte proliferation after injury, and that tenascin- $C$ is sufficient to induce proliferation in vitro. In the newt and zebrafish, ECM components and ECM-modifying proteases are among the most robustly enriched genes expressed in response to local injury; in contrast, in adult mammalian myocardium, inflammation and metabolic genes comprise the most significantly enriched transcripts. Over several weeks following injury, regeneration-competent hearts marginalize fibrotic ECM deposition to the periphery and replace it with regenerated myocardium, as observed in newts and zebrafish.

ECM components secreted from embryonic fibroblasts include fibronectin, collagen and heparin-binding EGF-like growth factor, and these factors can promote cardio-myocyte proliferation in a paracrine fashion. Therefore, it is possible that post-natal changes to ECM composition alter the proliferative capacity of cardio-myocytes. Studies in zebrafish have revealed that epicardium is especially important for initiating extracellular matrix deposition: injury in the myocardium induces epicardial cells to express fibronectin paralogues, Fn1 and Fn1b, within a day of injury. Through genetic ablation of fn1, Wang et al showed that fibronectin is required for heart regeneration, although not through the regulation of cardiomyocyte proliferation. Periostin is another secreted extracellular matrix component that has been shown to increase cardio-myocyte cell cycle activity. While in development periostin plays a role in epithelial mesenchymal transition (EMT), periostin is expressed in adult myocardium upon injury). Promoting collagen cross-linking in the ECM, periostin accelerates fibrillogenesis and contributes to scar formation. In a study by Kühn et al using the adult mouse MI model, exogenous expression of periostin enhanced post-injury myocardial proliferation and promoted cardiac repair, which resulted in improvement in ventricular remodeling and function. Periostin function was mediated through activation of $\beta$ integrins on cardio-myocytes; in its absence, activation of phosphatidylionsitol3-OH kinase was sufficient for cell cycle reentry. Controversy arose in the field when genetic manipulation of periostin did not alter cell cycle activity, cardio-myocyte content or cardiac repair in mice, in either ablation or overexpression.

Cell type composition also appears to have importance in the heart regenerative response due to their influence on the extracellular matrix. Fibroblasts greatly influence the composition of ECM deposition, and their presence has been shown to impact the proliferative capacity of cardio-myocytes in vitro. Fibroblasts are more abundant in the adult mammalian heart compared to fetal mammalian or adult non-mammalian hearts. Thus, changes in fibroblast abundance or composition may be responsible for the permissive versus non-permissive cardiac environment for regenerative growth.

Development vs. Regeneration in the heart: Transcriptional profile of cardio-myocytes in development and regeneration. A common principle observed in tissue regeneration is the reactivation of the previously employed developmental transcriptional programs. Studies in different model organisms have shown that mechanisms in developmental cardio-genesis 
also govern morphological regeneration of the injured heart. As discussed previously, in lower vertebrates and mammals, the source of new cardio-myocytes appears to be predominantly preexisting cardio-myocytes that re-enter the cell cycle, although a role for progenitors and stem cells remains hotly debated in the cardiovascular community.

The cardio-myocyte lineage originates from mesodermal cells that express T-box transcription factor Eomes and Mesp1, which is thought to be a regulator of cardiac progenitor cell fate. These precursors are later allocated to two major populations designated as the first heart field and the second heart field, defined by their crescent shapes distinct on day E7.5 in mouse embryo-genesis. As the heart tube forms and then loops to form the chambers, molecular cues further induce and define mesodermal progenitors to different cardiac cell types. An intricate gene regulatory network refines progenitor boundaries, terminal differentiation and transcriptional identities specific to each cell type during heart organogenesis. At early stages of development, NKX2-5 and ISL1 expression define cardiac progenitor cells; Ehrman This is followed by cardio-myocyte specific expression of Hopx in a subset of progenitors. Hopx defines cardio-myocyte cell fate and coordinates an antagonistic crosstalk between BMP and WNT pathways by physically interacting with BMP effector SMADs to repress WNT genes and promote cardiomyogenesis. During cardio-myocyte differentiation, zinc finger transcription factor GATA4 has an essential role in regulation of structural genes including $\alpha$-myosin heavy chain $(\alpha-\mathrm{mhc})$ and cardiac troponin C. Elucidating transcriptional profile of cardiac progenitors has enabled derivation of cardio-myocytes from embryonic stem cells, and their therapeutic potential is being tested in grafts into non-human primate models.

Reactivation of a GATA4-driven gene expression program plays a role in the context of regeneration as well consistent with the observation that the tissue activates an embryonic differentiation program for regenerating cardio-myocytes that acquire less organized sarcomeric structures Another developmental gene, Hand2, induces cardio-myocyte proliferation during regeneration Homeo-domain transcription factor MEIS1, which is required for normal cardiac development, has been shown to orchestrate postnatal cell cycle arrest and maturation. Strikingly, deletion of Meis1 in the adult heart is sufficient to induce cardio-myocyte cell cycle re-entry in adult cardio-myocytes. In addition to activation of developmental programs, expression of cardiac muscle genes and dedifferentiation facilitate mitotic and morphogenetic activity during post-injury response in newt regeneration. Dedifferentiation has also been explored in mammalian cardio-myocytes and many laboratories are now trying to dissect the transcriptional program that renders adult mammalian cardio-myocytes dedifferentiated sufficiently to be more permissive for cell cycle reentry. Recently, an analysis of global transcriptional programs in mammalian cardio-myocyte differentiation and regeneration has revealed that the regenerating mouse heart reverses the transcriptional processes of cardio-myocyte differentiation, with reactivation of latent developmental programs. Disruption of miRNA machinary in development and homeostasis results in cardiac abnormalities A role for microRNAs (miRNA) in cardio-myocyte proliferation and regeneration was revealed by numerous studies including a study by Porrello et al in 2011 and a fluorescent microscopy based screen MiRNAs are short RNA sequences that base-pair partially with messenger RNAs of target genes and thereby regulate gene expression. To date, dozens of miRNAs have been identified to play critical roles in not just cardio-myocyte DNA synthesis and cytokinesis but also post-natal mitotic arrest.Epigenetic control of cardio-genesis has also gained considerable attention, particularly because epigenetic events regulate maintanence of the proliferative state of cardio-myocytes through several mechanisms such as DNA methylation, chromatin remodeling, or covalent histone modifications including acetylation and methylation. The chromatin remodeling BAF complex and its subunit Baf60c have specific roles for transcriptional regulation at cardiac specific enhancer sites, and thus are essential for cardiac morphogenesis. Deletion of histone deacetylase Hdac2 with Hopx during cardiogenesis results in increased cardio-myocyte proliferation, and this effect is mediated through de-acetylation of Gata4.

Histone acetyl-transferase P300 directly regulates cardiac specification through Gata4, Mef2c and Srf.

Mice null for histone methyltransferase Smyd1 are embryonic lethal, and mice deficient in histone de-methylase Jarid2 die immediately after birth. Interestingly, JARID2 directly represses Notch target genes, thereby changing responsiveness of cells to the signaling events in the heart. Genetic deletion of Brahma-related gene 1, an ATP-dependent chromatin-remodeling factor, results in proliferation defects in myocardium. Thus, epigenetic events in the heart are dynamic and directly control cardio-myocytes and their proliferative state. Manipulating the epigenetic state of cardiomyocytes during the critical post-injury period is an attractive regenerative strategy, and histone de-acetylase inhibitors are potential therapeutic agents [23]."

Experimental project hypothesis: In order to verify finalistic or afinalistic characteristics of regenerative ability of some metazoan vs pathological process like neoplasia, fibrosis, or iperactivation of immune system is interesting observe "In a time related" way the kinetics of this 2 kind of process, observing the time that this process stop. Experimental project to be perfomed using invertebrates and various vertebrates models. At the end of the project it must be added comment related finalistic or a-finalistic process. Also interesting is to observe the genes involved and conservation or not between the transition from invertebrates to vertebrates and the phenotype expressed from embrio or adult form of species that not regenerate in the adult phases.

\section{Discussion}

Regenerative property of some metazoan species imply a fine regulation by genes to reproduce a specific part of body, without iper production of non useful tissue. This ability is showed more often in invertebrates. Is possible that this phenomena can be useful to better understand some pathological phenomena like 
Fibrosis or neoplasia or other in mammalians? In the transition from invertebrated to vertebrates is possible to verify that not increase gene but they are Re- arranged. Can be this fact to clear some pathological overproduction? The crucial fact is how can this kind of metazoan to reproduce correctly a normal part of the body? In regeneration of body part is an activity high regulated: in the right way without iper production of tissue and in a finalistic way [21-23].

And why this ability was loose in superior vertebrates? Or in some species limited in early stage of their life? What genes involved? Why there is a less frequence of neoplasia in invertebrates the vertebrates? and linke absent in echinoderm? Even malignancy of neoplasm differ from invertebrates to vertebrates: more metastatic properties in vertebrates. And related innate and adaptative immune response of invertebrates vs vertebtates show us somethings of relevant?

From reference is possible to verify that:

"The genetic programs which direct embryonic brain development are remarkably conserved and lends further support to the hypothesis that a common molecular bauplan underlies brain development in invertebrates and vertebrates" [1]. "Although similar numbers of ancestral genes have survived in each species, vertebrate lineages gained many more genes by duplication than invertebrate lineages, resulting in more than 200 homeobox genes in vertebrates and about 100 in invertebrates [2]". Related opioids receptors genes: "The quadruplication seems to coincide with, and, probably resulted from, the 2 proposed genome duplications in early vertebrate evolution. We conclude that the quartet of opioid receptors was already present at the origin of jawed vertebrates 450 million years ago [3]". "Regeneration is a trait that exists within different phyla, orders and species in the animal kingdom. Invertebrates such as planarians, crustaceans, cnidarians, echinoderms and insects, are known to have a strong global regenerative potential.

In amphibians, heart regeneration occurs during aquatic life but, to date, cardiac regeneration has not been documented in terrestrial amphibians. Cardiac regeneration in mammals appears to be restricted during a defined early-developmental period during embryonic and early-neonatal life. it is unclear whether the lack of regenerative potential in mammals is due to an improper re-activation of these developmental pathways or whether it is due to intrinsic cellular changes that prevent the cells from responding in a regenerative manner to the developmental cues (or both) non-cardiomyocyte populations in the heart also regenerate after myocardial damage and they are required to support cardio- myocyte proliferation and remodelling [4]". "Host responses against invading pathogens are basic physiological reactions of all living organisms. Since the appearance of the first eukaryotic cells, a series of defense mechanisms have evolved in order to secure cellular integrity, homeostasis, and survival of the host. Invertebrates, ranging from protozoans to metazoans, possess cellular receptors, which bind to foreign elements and differentiate self from non-self. This ability is in multicellular animals associated with presence of phagocytes, bearing different names (amebocytes, hemocytes, coelomocytes) in various groups including animal sponges, worms, cnidarians, mollusks, crustaceans, chelicerates, insects, and echinoderms (sea stars and urchins).these cells have a macrophage-like appearance and function and the repair and/ or fight functions associated with these cells are prominent even at the earliest evolutionary stage. The cells possess pathogen recognition receptors recognizing pathogen-associated molecular patterns, which are well-conserved molecular structures expressed by various pathogens (virus, bacteria, fungi, protozoans, helminths) [5]".

"Several features of amphioxus including the body plan, arrangement of hox genes, synteny of neighboring genes and embryonic development are more similar to the vertebrates than those same characteristics in tunicates [6]". "DNA methylation is evolutionarily conserved. Vertebrates exhibit high, widespread DNA methylation whereas invertebrate genomes are less methylated, predominantly within gene bodies. DNA methylation in invertebrates is associated with transcription level, alternative splicing, and genome evolution, but functional outcomes of DNA methylation remain poorly described in lophotrochozoans [7]".

"From yeasts to vertebrates, in order of increasing genome size and general biological complexity, increasing fractions of conserved bases are found to lie outside of the exons of known protein-coding genes. In all groups, the most highly conserved elements (HCEs), by log-odds score, are hundreds or thousands of bases long. These elements share certain properties with ultra conserved elements, but they tend to be longer and less perfectly conserved, and they overlap genes of somewhat different functional categories. In vertebrates, HCEs are associated with the 3' UTRs of regulatory genes, stable gene deserts, and megabase-sized regions rich in moderately conserved noncoding sequences. Noncoding HCEs also show strong statistical evidence of an enrichment for RNA secondary structure [8]“.

"During chordate evolution, we have searched for conserved elements in tunicate and vertebrate (Olfactores) genomes. We identified, for the first time, 183 non-coding sequences that are highly conserved between the 2 groups. Our results show that all but one element are conserved in non-syntenic regions between vertebrate and tunicate genomes, while being syntenic among vertebrates. in all the groups, they are significantly associated with transcription factors showing specific functions fundamental to animal development, such as multicellular organism development and sequence-specific DNA binding [9]”. “comparable developmental genetic mechanisms appear to operate in invertebrates and vertebrates during all major stages of brain development from the early specification of the neuroectoderm to the formation of neural circuitry [10]".

"Vertebrates diverged from other chordates B500 Myr ago and experienced successful innovations and adaptations, butthe genomic basis underlying vertebrate origins are not fully understood. Here 
we suggest, through comparison with multiple lancelet (amphioxus) genomes, that ancient vertebrates experienced high rates of protein evolution, genome rearrangement and domain shuffling and that these rates greatly slowed down after the divergence of jawed and jawless vertebrates. Compared with lancelets, modern vertebrates retain, less protein diversity, fewer nucleotide polymorphisms, domain combinations and conserved non-coding elements (CNE). Modern vertebrates also lost substantial transposable element (TE) diversity, whereas lancelets preserve high TE diversity that includes even the long-sought RAG transposon. Lancelets exhibit rapid gene turnover, pervasive transcription, fastest exon shuffling in metazoans and substantial TE methylation not observed in other invertebrates [11]".

"The origin of vertebrates and of jawed vertebrates is characterized by a doubling of the vertebrate genome, leading to hypothesesthat this genomic event drove organismal macroevolution [12]". "At morphological and functional level, invertebrate telocytes from the body of leech Hirudo medicinalis (Annelida), suggesting how these cells, forming a resident stromal 3D network, can influence or participate in different events. leech telocytes: i) are organized in a cellular dynamic and versatile 3D network likewise the vertebrate counterpart; ii) are an evolutionarily conserved immune-neuroendocrine system; iii) form an immuno-surveillance system of resident cells responding faster than migrating immunocytes recruited in stimulated area; iv) communicate with neighbouring cells directly and indirectly, via cell-cell contacts and soluble molecules secreted by multi vesicular bodies; v) present within neo-vessels, share with immunocytes the mesodermal lineage; vi) are involved in regenerative processes. we propose that HmTCs, integrating so different functions, might explain the innate immune memory and can be associated with several aged related diseases $[13,14]^{\prime \prime}$.

"Cardiac fibrosis is implicit inall forms of heart disease. Fibrosis is a scarring process characterised by myo fibroblast accumulation and excessive deposition of extracellular matrix (ECM) proteins, inparticular collagen I and III. This can lead to loss of organ architecture and compliance, induction of pathological signalling in cardio -myocytes and eventual heart failure [15]". "Exposure to crystalline silica particles causes silicosis, an occupational disease leading to an overproduction of collagen in the lung. The first step of this pathology is characterized by the release of inflammatory mediators. Tumour necrosis factor (TNF) is a pro-inflammatory cytokine directly involved in silica-induced pulmonary fibrosis. The marine demosponge Chondrosia reniformis is able to incorporate silica grains and partially dissolve the crystalline forms apparently without toxic effects. In the present work C. reniformis tissue explants were treated with fine quartz dust and the expression level of fibrogenic genes was assayed by qPCR, demonstrating an overexpression of a fibrillar and a non-fibrillar collagen and of prolyl-4-hydroxylase enzyme. The deposition of new collagen could also be documented in quartz-treated sponge explants [16]".

"The term "macrophage" (phagocyte) was coined by Ilya Ilyich Mechnikov, a comparative embryologist and winner of the Nobel
Prize in 1908. He observed in starfish larvae a group of cells that had unusual characteristics - the ability to move in tissue: after introducing small rose thorns into the larvae, he noted the next morning that the thorns were surrounded by the mobile cells. He further studied this phenomenon using the freshwater fl ea (Daphnia magna), exposing itto fungal spores: the spores were attacked and isolated by the Daphnia macrophages. Macrophagelike cells are present in many species of invertebrates. Often they originate from mesenchymal, endothelial, or fibroblastic cells that differentiate into phagocytes For example, Hydra, a member of the phylum Cnidaria, has cells with phagocytic capability that play a role in the animal's ability to recognize "self" and, thus, make it a useful model of graft rejection. Molluscs also have cells that can act as macrophages, but the origin of these cells is mesenchymal, not hematopoietic, indicating divergent paths of differentiation" [17].

"Immunology deals with the typical strategies of biological defence against environmental toxins and pathogens. Components of immune system and their coherent functional attributes enabled the invertebrates to overcome the toxin-induced chemical stresses of the primitive and modern global environment. Many of the environmental pollutants of the current hydrosphere are less researched, with limited toxicological information. Invertebrates over a period of time are assumed to evolve novel and unique modes of immunological reactivities to defend against the toxic insults of environmental pollutants at the cellular and molecular levels. Aquatic invertebrates are relatively a neglected group of organisms with reference to their immune toxicological status in a bioun safe environment. The principal immune toxicological responsiveness of invertebrates centre around the reactivities of circulating immunocytes and selected effector organs like gill, digestive gland, intestine, labial palp" [18].

"Itis known thatneoplasia is a pathological process characterized by an overgrowth of a new tissue in the context of a pre-existing one, and consists of atypical cells, a term which incorporates the sum of the differences in morphological, biochemical and functional features of cancer cells relative to normal cells. neoplastic tissue is characterized by a self-growing, progressive, irreversible and nonfinalistic behavior.2 predominant types of neoplasia have been reported in marine molluscs, viz. disseminated neoplasia, also called leukaemia or hemic neoplasia (HN), andgonadal neoplasia [19]." "The ability to demonstrate metastasis in an invertebrate is a difficult problem for several reasons: (1) many invertebrates have no circulatory system (2) the majority of invertebrates that do have a circulatory system is based on an open system with large blood sinuses (3) many invertebrates have non discrete organ systems (4) relatively few neoplasms have been reported in invertebrates and (5) the majority of neoplasms that have been described are anaplastic in appearance [20]".

"Generally accepted assumption that malignancies with metastasis or true cancers are tightly linked to the adaptive immune system of jawed vertebrates, and the corollary that associates the lack of adaptive immunity in invertebrates with the rarity and benignity of neoplasia are perhaps a bit too premature 
and simplistic.it could be useful to reevaluate the available evidence of tumor occurrence and severity in invertebrates and jawless vertebrates whose immune systems are fundamentally different from those of mammals [21]." So the regenerative abilities of metazoa depends on a specific program genetically controlled so the loose of this ability can be due to different facts:

1) The complete performing of the program with complete reproduce -regeneration of a body part genetically controlled in some kind of species orin determinate stage of life.

2) A genetically signal that stop this process in some metazoa or in determinante stage of their life (in examples adults vs embryo).

3) A loose of a sort of "growing" regenerative signal genetically controlled.

\section{4) Undetermined}

\section{Conclusion}

Related the reference reported in this work and to the experimental project hypotesys to be tested is possible to say that some conserve genes between invertebrates and vertebrates responsible of regeneration of body part (regular) must be deeply investigated to verify why in some condition in superior vertebrates some phenomena over produce pathological damages instead to stop. Relevant the gene involved in this properties. Interesting information can be produced in field of pathology, immunology and oncology. Afinalistic Hyper production of fibrotic tissue, or neoplasia or hyper activation of some line of immune systems (innate or adaptative). Differ from a regular regenerative process (finalistic process) but the genes involved in this similar process must be of same origin and this facts must be deeply investigated.

The fact that regenerative properties in some metazoan are limited to early stage of life is very interesting phenomena: a control of the system that can be interesting to observe also to control some pathology with iperproduction like neoplasm, fibrosis, and some immune disease.

\section{Clarifications}

This work is produced without any diagnostic or therapeutic intent only to submit to the researcher new Hypotesis to verify.

\section{Acknowledgement}

None.

\section{Conflict of Interest}

No conflict of interest.

\section{References}

1. Kammermeier L, Reichert H (2001) Common developmental genetic mechanisms for patterning invertebrate and vertebrate brains. Brain Res Bull 55(6): 675-682.

2. Nam J, Nei M (2005) Evolutionary change of the numbers of homeobox genes in bilateral animals. Mol Biol Evol 22(12): 2386-2394.
3. Susanne Dreborg, Gorel Sundstrom, Tomas A Larsson, Dan Larhammar (2008) Evolution of vertebrate opioid receptors; In: Tomas Ho" kfelt (Ed.), Karolinska Institutet, Stockholm, Sweden.

4. Vivien CJ, Hudson JE, Porrello ER (2016) Evolution, comparative biology and ontogeny of vertebrate heart regeneration. NPJ Regen Med 1: 16012.

5. Buchmann K (2014) Evolution of Innate Immunity: Clues from Invertebrates via Fish to Mammals. Front Immunol 5: 459.

6. Graeme J Roch, Javier A Tello, Nancy M Sherwood (2014) At the Transition from Invertebrates to Vertebrates, a Novel GnRH-Like Peptide Emerges in Amphioxus. Mol Biol Evol 31(4): 765-778.

7. Guillaume Rivière (2014) Epigenetic features in the oyster Crassostrea gigas suggestive of functionally relevant promoter DNA methylation in invertebrates. Front Physiol 5:129.

8. Adam Siepel, Gill Bejerano, Jakob S Pedersen, Angie S Hinrichs, Minmei Hou, et al. (2005) Evolutionarily conserved elements in vertebrate, insect, worm, and yeast genomes. Genome Res 15(8): 1034-1050.

9. Remo Sanges, Yavor Hadzhiev, Marion Gueroult-Bellone, Agnes Roure, Marco Ferg, et al. (2013) Highly conserved elements discovered in vertebrates are present in non-syntenic loci of tunicates, act as enhancers and can be transcribed during development. Nucleic Acids Res 41(6): 3600-3618.

10. Heinrich Reichert (2009) Evolutionary conservation of mechanisms for neural regionalization, proliferation and interconnection in brain development. Biol Lett 5(1): 112-116.

11. Shengfeng Huang, Zelin Chen, Xinyu Yan, Ting Yu, Guangrui Huang, et al. (2014) Decelerated genome evolution in modernvertebrates revealed by analysis of multiplelancelet genomes. Nat Commun 5: 5896.

12. Philip CJ Donoghue, Mark A Purnell (2009) The Evolutionary Emergence of Vertebrates From Among Their Spineless Relatives. Evo Edu Outreach 2: 204-212

13. Chan ET1, Quon GT, Chua G, Babak T, Trochesset M, et al. (2009) Conservation of core gene expression in vertebrate tissues. J Biol 8(3): 33.

14. Pulze L, Capri M, Grimaldi A, Salvioli S, Tettamanti G, et al. (2018) A new cellular type in invertebrates: first evidence of telocytes in leech Hirudo medicinalis. J Immunol Sci 2(1): 22-25.

15. Wang Z, Stuckey DJ, Murdoch CE, Camelliti P, Lip GYH, et al. (2018) Cardiac fibrosis can be attenuated by blocking the activity of transglutaminase 2 using a selective small-molecule inhibitor. Cell Death and Disease 9: 613.

16. Pozzolini M, Scarfi S, Gallus L, Ferrando S, Cerrano C, et al. (2017) Silicainduced fibrosis: an ancient response from the earlymetazoans. Journal of Experimental Biology 220: 4007-4015.

17. Wilson-Sanders SE (2011) Wilson-Sanders Invertebrate Models for Biomedical Research, Testing, and Education 126. ILAR Journal 52(2): 126-152.

18. Sajal Ray, Soumalya Mukherjee, Niladri Sekhar Bhunia, Anindya Sundar Bhunia, Mitali Ray (Year) Immunotoxicological Threats of Pollutants in Aquatic Invertebrates. http://dx.doi.org/10.5772/60216 INTECH open science.

19. De Vico G, Carella F (2015) Tumors in invertebrates: molluscs as an emerging animal model for human cancer. ISJ 12: 19-21.

20. Keith R Cooper (In press) Metastasis of Invertebrate Neoplasms (chapter) in book: Comparative Aspects of Tumor Development, pp. 3738.

21. Jacques Robert (2010) Comparative study of tumorigenesis and tumor immunity in invertebrates and vertebrates and nonmammlian vertebrates. Dev Comp Immunol 34(9): 915-925.

22. Daniela Melillo, Rita Marino, Paola Italiani, Diana Boraschi (2018) Innate Immune Memory in Invertebrate Metazoans: A Critical Appraisal. Front Immunol 9: 1915

23. Aysu Uygur, Richard T Lee (2016) Mechanisms of Cardiac Regeneration. Dev Cell 36(4): 362-374. 